\title{
Speckle Reduction for Ultrasonic Imaging Using Frequency Compounding and Despeckling Filters along with Coded Excitation and Pulse Compression
}

\author{
Joshua S. Ullom, ${ }^{1}$ Michael L. Oelze, ${ }^{2}$ and Jose R. Sanchez ${ }^{3}$ \\ ${ }^{1}$ Harris Corporation, Melbourne, FL 32905, USA \\ ${ }^{2}$ Electrical and Computer Engineering Department, University of Illinois at Urbana-Champaign, Urbana, IL 61801, USA \\ ${ }^{3}$ Electrical and Computer Engineering Department, Bradley University, Peoria, IL 61625, USA
}

Correspondence should be addressed to Jose R. Sanchez, jsm@bradley.edu

Received 27 November 2011; Accepted 5 April 2012

Academic Editor: Erdal Oruklu

Copyright ( $) 2012$ Joshua S. Ullom et al. This is an open access article distributed under the Creative Commons Attribution License, which permits unrestricted use, distribution, and reproduction in any medium, provided the original work is properly cited.

\begin{abstract}
A method for improving the contrast-to-noise ratio (CNR) while maintaining the $-6 \mathrm{~dB}$ axial resolution of ultrasonic $\mathrm{B}$ mode images is proposed. The technique proposed is known as eREC-FC, which enhances a recently developed REC-FC technique. REC-FC is a combination of the coded excitation technique known as resolution enhancement compression (REC) and the speckle-reduction technique frequency compounding (FC). In REC-FC, image CNR is improved but at the expense of a reduction in axial resolution. However, by compounding various REC-FC images made from various subband widths, the tradeoff between axial resolution and CNR enhancement can be extended. Further improvements in CNR can be obtained by applying postprocessing despeckling filters to the eREC-FC B-mode images. The despeckling filters evaluated were the following: median, Lee, homogeneous mask area, geometric, and speckle-reducing anisotropic diffusion (SRAD). Simulations and experimental measurements were conducted with a single-element transducer $(f / 2.66)$ having a center frequency of $2.25 \mathrm{MHz}$ and a $-3 \mathrm{~dB}$ bandwidth of 50\%. In simulations and experiments, the eREC-FC technique resulted in the same axial resolution that would be typically observed with conventional excitation with a pulse. Moreover, increases in CNR of 348\% were obtained in experiments when comparing eREC-FC with a Lee filter to conventional pulsing methods.
\end{abstract}

\section{Introduction}

In imaging, the ability to detect small or low-contrast structures is of utmost importance. However, ultrasonic images are riddled with speckle, which reduces the ability to detect low-contrast and/or small-sized targets. Speckle is formed by subresolution scatterers that cause constructive and destructive interference of backscattered ultrasonic signals within the resolution cell volume of an ultrasonic source [1]. In ultrasound, the difference in contrast between different soft tissues could be as small as $1 \%$. Consequently, speckle reduction techniques must be applied to improve image contrast and enhance the detectability of structures having low contrast with the background [2].

Speckle-reduction techniques can be classified into two categories: compounding methods and postprocessing techniques. The compounding speckle-reduction methods include spatial [3-6] and frequency compounding [7-11]. These schemes rely on making separate images that have uncorrelated or partially correlated speckle patterns. These images are then averaged to reduce the speckle but at the expense of spatial resolution. Postprocessing specklereduction techniques [12-17] reduce speckle after the ultrasound image is formed. The engineering tradeoffs vary based on the postprocessing speckle-reduction technique employed but typically include increased contrast and reduced speckle versus edge preservation, image blurring, and image texture.

A recently developed speckle-reduction techniqueresolution enhancement compression with frequency compounding (REC-FC) — can improve the visibility of ultrasonic images while extending the tradeoff between spatial 
resolution and visibility $[18,19]$. REC-FC used the coded excitation and pulse compression technique, REC, which has the potential to improve the axial resolution of an ultrasonic imaging system by a factor of two [20]. A larger axial resolution translated into a larger bandwidth. In addition to increases in bandwidth, the REC technique has the typical benefits of coded excitation and pulse compression such as increased time bandwidth product (TBP) [21]. An excitation signal with a longer duration than a conventional excitation signal contains more energy, resulting in an increased echo signal-to-noise ratio (eSNR) [22]. Consequently, increased eSNR results in deeper penetration depth. However, because the pulse duration is increased, the axial resolution degrades. To restore the axial resolution, pulse compression techniques, such as a Wiener filter, are used [21].

In REC, the larger bandwidth was exploited by combining the technique with FC. FC is a speckle reduction technique that subdivides the spectrum of the radio-frequency (RF) echoes into subbands to make partially uncorrelated images [7]. These images were then compounded to reduce the speckle variance. $\mathrm{REC}-\mathrm{FC}$ was found to improve contrast-to-noise ratio (CNR) by as much as $231 \%$ compared to a conventional pulsing (CP) scheme. Overall, RECFC improved image quality, CNR, and lesion boundaries. However, the drawback of REC-FC was that subband filters only contained a fraction of the original system bandwidth, which resulted in a reduction of axial resolution.

In this study, an improvement to the REC-FC technique is proposed, which enhances the visibility of an ultrasonic image while maintaining the axial resolution to comparable levels when exciting a transducer with a pulse. The resulting image can be further enhanced by reducing the speckle and improving the visibility by applying postprocessing despeckling filters.

\section{Methods and Procedures}

2.1. REC. In REC, a preenhanced chirp, $x(t)$, is used to excite an ultrasonic focused source, $h(t)$. The preenhanced chirp is obtained through convolution equivalence as discussed in $[18,20]$. The goal of the preenhanced chirp is to boost the energy in the band edges of the source's frequency response.

With REC, the spectral support of the echo signal is much larger than the bandwidth of the source. However, the pulse duration of the excitation signal, $y(t)$, is longer than an echo from the same source when the excitation is $\delta(t)$, which will be described as conventional pulsing (CP) in this study. Therefore, in order to recover the benefits of the larger bandwidth, the resolution must be restored through pulse compression. Pulse compression is performed using a Wiener filter, which is described by [21]:

$$
H_{\text {wiener }}(f)=\frac{\Psi^{*}(f)}{|\Psi(f)|^{2}+\gamma \overline{\operatorname{eSNR}(f)}-1},
$$

where $f$ is frequency, and $\gamma$ is a smoothing parameter that controls the tradeoff between sidelobe levels, axial resolution, and eSNR. The term $\overline{\mathrm{eSNR}}$ is the echo signalto-noise ratio per frequency channel and $\Psi(f)$ corresponds to the Fourier transform of a linear chirp excitation, which is part of convolution equivalence scheme used to obtain the preenhanced chirp as discussed in $[18,20]$. In practice, the eSNR is estimated for the imaging system by pointing the imaging system to a region where no scatterers exist, for example, water, and the noise of the system can be isolated for a particular excitation. The compressed echo waveform and the log-compressed envelope of the echo waveform for a point target are shown in Figure 1 along with the CP reference. The Wiener filter allows the compression to balance between matched filtering and inverse filtering. Matched filtering provides the best gain in SNR but results in larger sidelobes and loss in axial resolution. An inverse filter provides the best compression terms of axial resolution and sidelobes but amplifies noise in the system.

2.2. REC-FC. In REC-FC, the wideband RF spectrum of each scan line was partitioned into $N$ subbands by using Gaussian bandpass filters. These Gaussian bandpass filters contained a fraction of the original system bandwidth. The resulting images from the $N$ subbands were compounded to form an image with reduced speckle variance. A reduction in speckle variance translated into CNR improvements. However, because the subband width was smaller in bandwidth than the original system, the axial resolution in the compounded images deteriorated. For example, with REC the axial resolution is doubled compared to CP. If overlapping subbands with a width of half the REC bandwidth (full width) are applied, then the resulting axial resolution is the same as CP and the image has improved visibility because of the compounding effects. The tradeoff of axial resolution versus image visibility is shown in Figure 2 for various subband widths. All subband widths are compared to CP, for example, third width implies that subbands with one-third of the CP bandwidth are applied and then compounded.

2.3. Enhanced REC-FC. In this study, a method is proposed that could provide the improvements in visibility that were obtained with REC-FC but without degrading the $-6 \mathrm{~dB}$ axial resolution beyond the axial resolution obtained for CP. The proposed method consists of compounding RECFC images obtained from different subband widths, which will reduce the speckle variance even further and result in an improvement of image visibility. This technique will be known as enhanced REC-FC or eREC-FC [23]. Moreover, the method has no impact on the lateral resolution of the imaging system. In this study, eREC-FC utilized the uniformly weighted sum of the following images (Figure 2): REC reference image, REC-FC (full-width), REC-FC (halfwidth), REC-FC (third-width), REC-FC (fourth-width), and REC-FC (eighth-width) to form a final enhanced REC-FC image. The original REC image was included because the borders of the lesion in the eREC-FC image become much more distinct because of the high spatial resolution of the REC technique. REC-FC (eight-width) was the final image compounded because smaller subband widths require too much computation time for the minimal improvements in CNR. Combining REC with REC-FC (eighth-width) resulted 


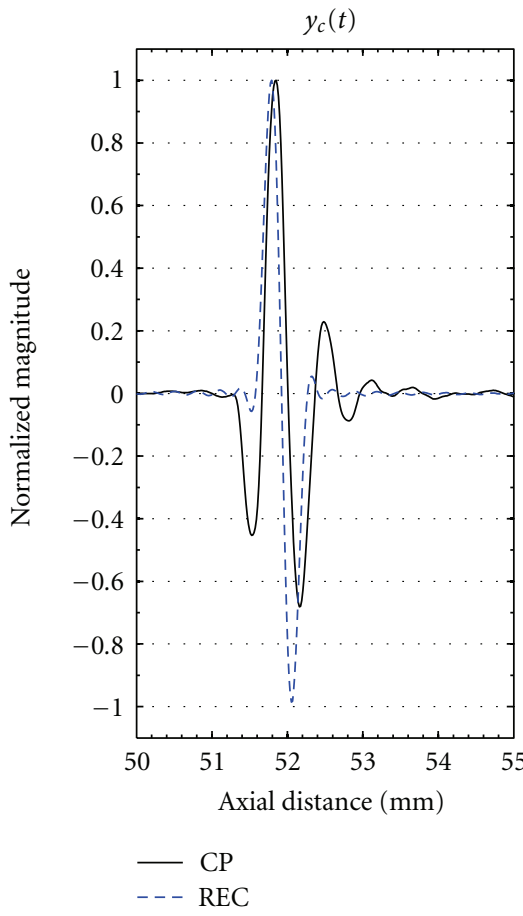

(a)

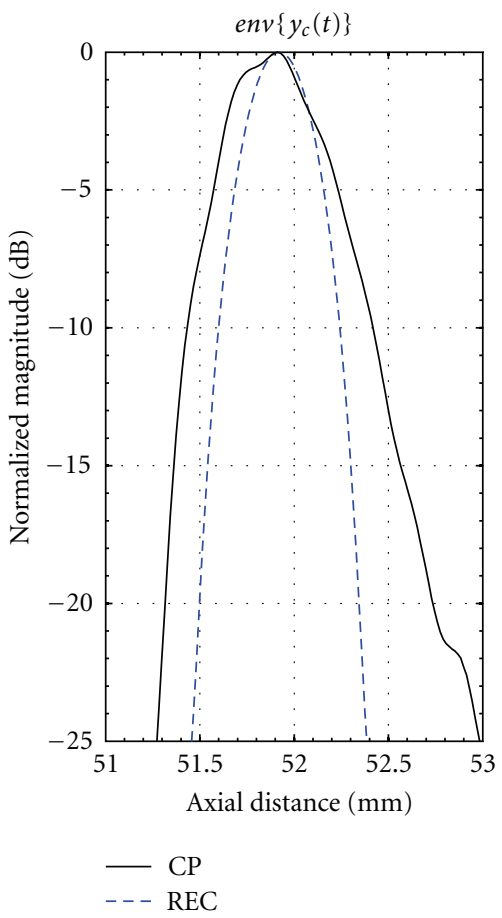

(b)

FIGURE 1: Compression of the $y(t)$ is represented by $y_{c}(t)$ and the log-compressed and envelope-detected version of $y_{c}(t)$.

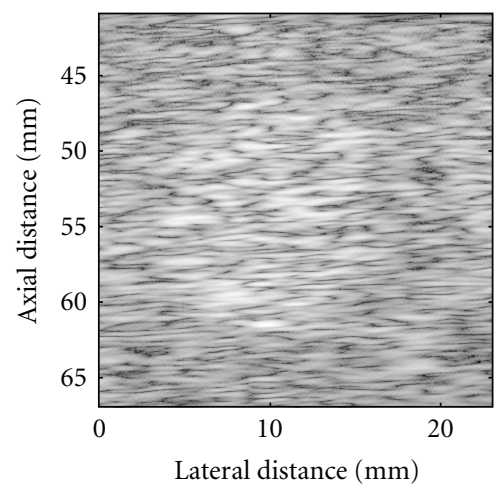

(a)

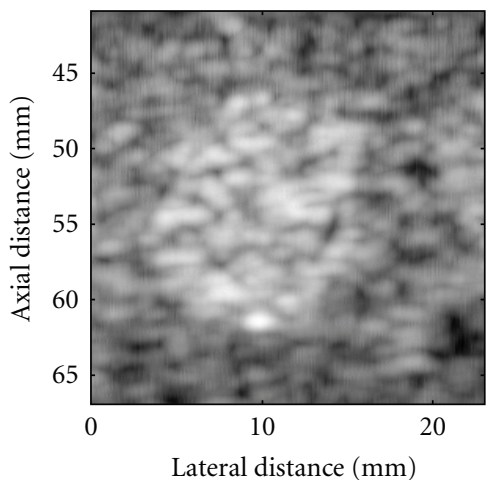

(d)

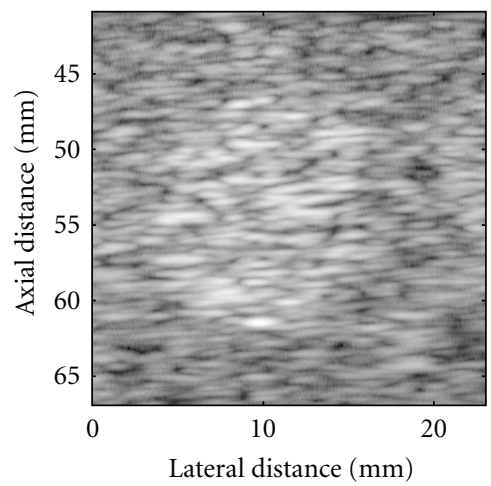

(b)

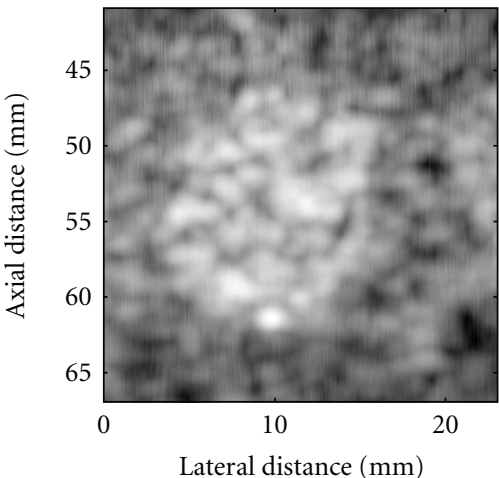

(e)

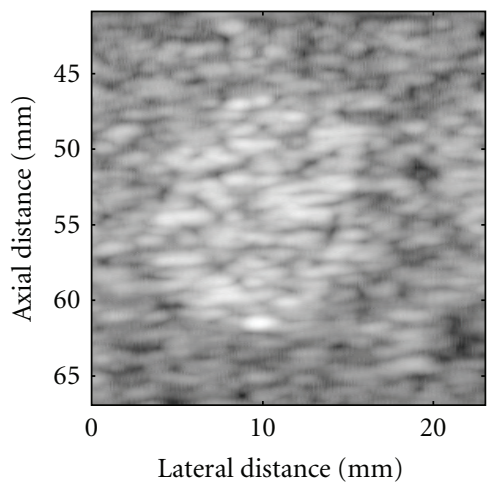

(c)

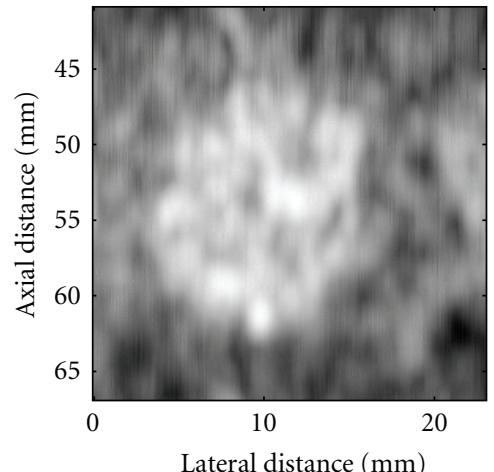

(f)

Figure 2: B-mode images for (a) REC reference, (b) REC-FC (full-width), (c) REC-FC (half-width), (d) REC-FC (third-width), (e) REC-FC (fourth-width), and (f) REC-FC (eighth-width). Image dynamic range is $-50 \mathrm{~dB}$. 
in similar CNR as in the eREC-FC technique presented herein; however, the spatial resolution in the image would be far worse than the eREC-FC technique. Therefore, by combining more images of varying subbands improvements in axial resolution could be obtained.

Theoretically, by summing all the compounded images along with the reference, the final enhanced image would have a $-6 \mathrm{~dB}$ axial resolution similar to the full-width REC-FC scenario or equivalent to the original resolution obtained with CP. The results of summing the envelope of the reference and subbands are shown in Figure 3. Evaluation of the eREC-FC envelope at $-6 \mathrm{~dB}$ in Figure 3(b) indicated that a loss of $10 \mu \mathrm{m}$ in axial resolution was obtained when compared to CP. Compared to the wavelength of the source, the loss is $1.5 \%$. Furthermore, every drop of $-6 \mathrm{~dB}$ in amplitude is followed by a slight deterioration in the axial resolution. However, this degradation should not affect the image quality unless there is a large contrast difference, such as in a cystic lesion (i.e., no scatterers).

2.4. Despeckling Filters. Images obtained with the eREC-FC technique were further processed with several despeckling filters. These techniques could also be applied to $\mathrm{CP}$ and REC excitations. However, the goal of this particular study was to judge how well image quality would be improved by applying coded excitation, novel compounding techniques, and postprocessing filters. Therefore, to better manage the amount of data for comparisons, only filtering techniques will be applied to the eREC-FC images. Similar improvements provided to eREC-FC by the filtering techniques are also expected for CP and simple REC excitations (except that the starting point for eREC-FC in terms of image quality is already improved leading to overall better improvement using filtering for eREC-FC). Despeckling filters make use of a moving, overlapping window of size $(n \times n)$, where $n$ is an odd integer, that advances through the entire image one pixel at a time. The center pixel of the window is the location that will be adjusted in the filtered image. Some despeckling filters use iterative techniques, where after the first iteration (filtering of the original image) the filtered image becomes the input to the filter for each successive iteration. The despeckling filters used in this study were as follows.

2.4.1. Median Filtering [12, 13]. Median filtering makes use of a moving, overlapping window. The median of the pixels in the window is the resulting value of the center pixel in the window for the filtered image. Median filtering is used to smooth an image and minimize or eliminate noise spikes, with the idea that all pixels in a small region of an image should be similar.

2.4.2. Lee Filtering [14]. Lee filtering also uses a moving, overlapping window. The Lee filter uses statistics within that window such as mean and variance to adjust the resulting center pixel of the window. The equation that governs this filtering process $[24,25]$ is

$$
f_{i, j}=\bar{g}_{i, j}+k_{i, j} \cdot\left[g_{i, j}-\bar{g}_{i, j}\right]
$$

where $i$ and $j$ are pixel coordinates, $f_{i, j}$ is the filtered pixel at location $(i, j), \bar{g}_{i, j}$ is the mean of the pixel intensities in the window, $g_{i, j}$ is the center pixel in the window, and

$$
k_{i, j}=\frac{1+\bar{g}_{i, j} \sigma^{2}}{\sigma^{2}\left(1+\sigma_{n}^{2}\right)},
$$

where $\sigma^{2}$ is the variance in the window and $\sigma_{n}^{2}$ is the noise variance in the whole image. This will result in $k \in[0,1]$. Because the variance in noise, or speckle, is not known, it is estimated by [24]

$$
\sigma_{n}^{2}=\sum_{I} \frac{\sigma_{w_{b}}^{2}}{\bar{w}_{b}}
$$

where $w_{b}$ is a window that is 10 times larger than the filtering window, and $\sigma_{w_{b}}^{2}$ and $\bar{w}_{b}$ are the variance and mean of pixel intensity of the larger window, $w_{b}$, respectively. This window moves through the entire image, $I$. Statistics obtained for each region are combined over the entire image to obtain a single estimate of speckle noise.

2.4.3. Homogeneous Mask Area Filtering [24, 26]. Two windows are used in the homogeneous mask area filtering technique, a large main window, which determines the pixel location to filter, and a smaller subwindow within the main window. For each subwindow, a speckle index is calculated as

$$
S=\frac{\sigma^{2}}{\mu},
$$

where $\mu$ and $\sigma^{2}$ are the mean and variance of the pixel intensity in the subwindow, respectively. The mean pixel intensity of the subwindow with the smallest speckle index becomes the filtered pixel value. For this study, the dimension of the subwindow was $(n-2) \times(n-2)$.

2.4.4. Geometric Filtering [27]. Geometric filtering uses a moving, overlapping window of size $3 \times 3$. In addition, the geometric filter uses an iterative approach to make the center pixel of the window more like its neighboring pixels. The idea behind the geometric filter is that a very small region of an image should be homogeneous. There are four directions the geometric filter iterates through north-south, east-west, northwest-southeast, and northeast-southwest. In each case, a line of three pixels is created and evaluated. The algorithm for computing the filtered pixel update is shown hereinafter. In the first iteration, $a$ would correspond to the pixel in the 


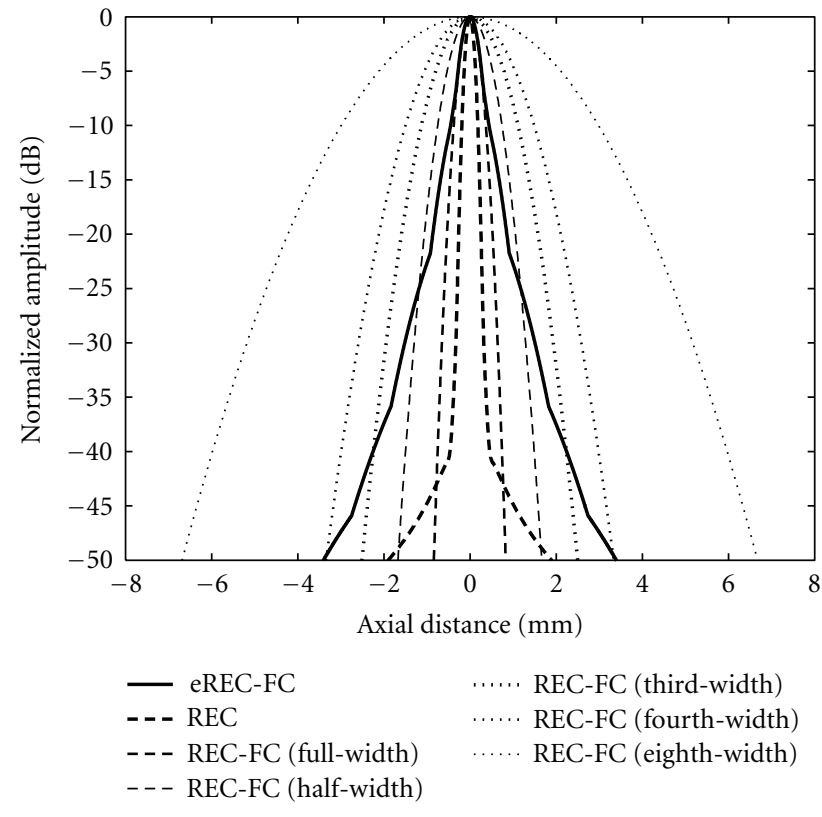

(a)

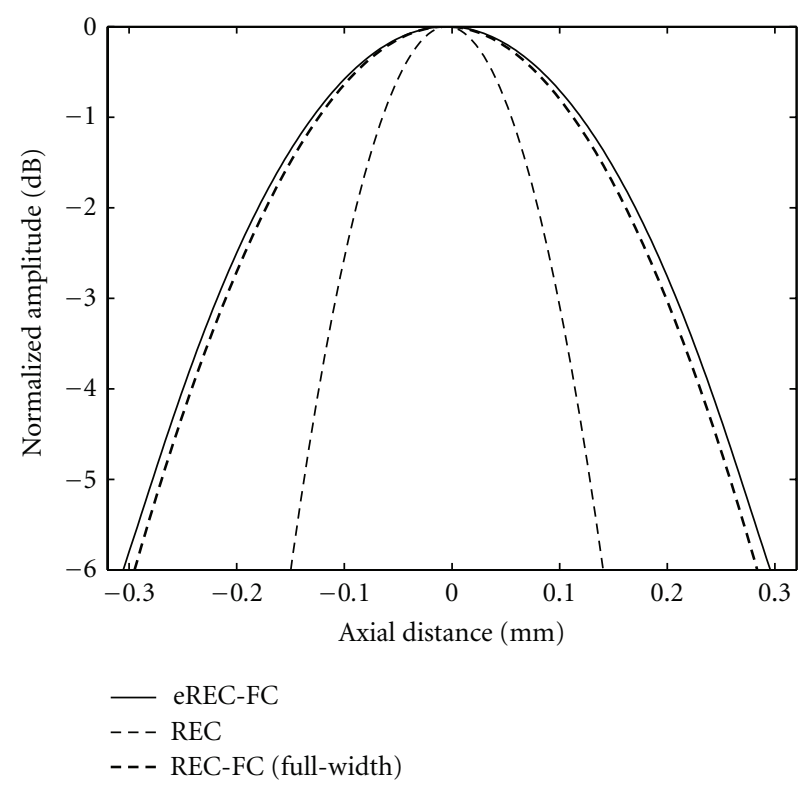

(b)

FIGURE 3: (a) Individual envelopes showcasing the axial resolution for the REC reference case. the REC-FC cases, and the eREC-FC case. (b) Zoomed version of eREC-FC showing that the axial resolution was similar to REC-FC (full-width). Note that the axial resolution for REC-FC (full-width) is the same as CP.

north direction, $b$ is the center pixel, and $c$ would correspond to the pixel in the south direction $[24,27]$ :

$$
\begin{aligned}
& \text { if } a \geq b+2, \text { then } b=b+1, \\
& \text { if } a>b \text { and } b \leq c \text {, then } b=b+1, \\
& \text { if } c>b \text { and } b \leq a \text {, then } b=b+1, \\
& \text { if } c \geq b+2, \text { then } b=b+1, \\
& \text { if } a \leq b-2, \text { then } b=b-1, \\
& \text { if } a<b \text { and } b \geq c \text {, then } b=b-1, \\
& \text { if } c<b \text { and } b \geq a \text {, then } b=b-1, \\
& \text { if } c \leq b-2, \text { then } b=b-1 .
\end{aligned}
$$

2.4.5. Speckle-Reducing Anisotropic Diffusion [16]. Specklereducing anisotropic diffusion (SRAD) is an algorithm that smears the pixel intensities within homogeneous regions while preserving edges by not smearing across inhomogeneous regions. SRAD is based on anisotropic diffusion [28] and is used by solving the diffusion equation described as a nonlinear partial differential equation:

$$
\begin{gathered}
\frac{\partial I}{\partial t}=\operatorname{div}[c(|\nabla I|) \cdot \nabla I], \\
I(t=0)=I_{0},
\end{gathered}
$$

where div is the divergence operator, $\nabla$ is the gradient operator, $I_{0}$ is the original image, and is greater than zero. $c$ is the instantaneous coefficient of variation and is described by

$$
c(x)=\frac{1}{1+(x / k)^{2}},
$$

where $x$ is a spatial position and $k$ is an edge magnitude parameter.

2.5. Image Quality Metrics. To evaluate the performance of the eREC-FC technique and the eREC-FC with despeckling filters compared with $\mathrm{CP}$ the following image quality metrics were used.

2.5.1. Contrast-to-Noise Ratio (CNR) [2]. CNR, also known as contrast-to-speckle ratio, is a quantitative measure that will assess image quality and describe the ability to perceive a target from the background region. CNR is defined as

$$
\mathrm{CNR}=\left|\frac{\mu_{B}-\mu_{T}}{\sqrt{\sigma_{B}^{2}+\sigma_{T}^{2}}}\right|,
$$

where $\mu_{B}$ and $\mu_{T}$ are the mean brightness of the background and the target lesion and $\sigma_{B}^{2}$ and $\sigma_{T}^{2}$ are the variance of the background and target, respectively. To avoid possible errors in the calculations due to attenuation, the evaluated regions of interest in the background and the target lesion will be of the same size and are located at the same depth. A larger CNR represents better contrast.

2.5.2. Histogram Pixel Intensity (HPI). HPI is the mean of the frequency distribution of gray-scale pixel intensities and is described by

$$
\mathrm{HPI}=E\{B\},
$$


where $B$ is the histogram being evaluated and is described by

$$
B(i)=c_{i}
$$

where $c_{i}$ represents the number of pixels in the image within a particular intensity level, $i$, which is an integer between 0 and 255 that represents the grayscale levels used in B-mode images. Histograms will be made for same-sized regions for the target lesion and the background and located at the same depth. Ideally, for superior target detectability, there is no overlap present between the target histogram and the background histogram. Therefore, histogram overlap ( $\mathrm{HO})$, the percentage of overlapping pixels between these two regions, will be considered as well. In addition to $\mathrm{HO}$, the difference between the distributions for mean pixel intensity for the target and the background will be quantified in order to assess the separation between both distributions. This quantity will be known as $H_{\text {diff }}$. Consequently, the technique with the least amount of overlap and the greatest separation would represent the technique with the best target detectability.

2.5.3. Margin Strength (MS). Estimates of MS [29] were used to detect the edges in the B-mode images. First, a thresholding scheme was applied to the images. Then, MS was estimated to detect the strength of the boundaries using the following expression:

$$
\mathrm{MS}=E\left\{\sqrt{\left(\frac{d \mathrm{ROI}}{d x}\right)^{2}+\left(\frac{d \mathrm{ROI}}{d y}\right)^{2}}\right\}
$$

where $E$ is the expectation operator, ROI is the region of interest within the envelope, and $x$ and $y$ correspond to the image coordinates. The margin strength is then imaged, which provides a mechanism to qualitatively study the edge of the targets being imaged.

2.5.4. Comparative Signal-to-Noise Ratio (cSNR) [24, 30]. cSNR is a comparative measure that quantifies the amount of noise/speckle reduction between the filtered and the unfiltered image. cSNR is described by

$$
\mathrm{cSNR}=10 \log _{10} \frac{\sum_{i=1}^{M} \sum_{j=1}^{N}\left(g_{i, j}^{2}+f_{i, j}^{2}\right)}{\sum_{i=1}^{M} \sum_{j=1}^{N}\left(g_{i, j}-f_{i, j}\right)^{2}}
$$

A larger cSNR represents a larger reduction of speckle noise. In this study, each filtered image, $g$, is compared to the reference image using $\mathrm{CP}, f$.

2.6. Computer Simulations. Computer simulations were carried out in MATLAB (MathWorks, Natick, MA) to characterize the performance of the eREC-FC technique along with the despeckling filters. The simulations used a received pulseecho pressure field model [31] described as

$$
g(x, y, t)=h_{1}(t) * f(x, y) * h_{\mathrm{pe}}(y, t),
$$

where $x$ represents the axial spatial coordinate, $y$ represents the lateral spatial coordinate, $h_{1}(t)$ is the pulse-echo impulse response of the transducer, $f(x, y)$ is the scattering function, and $h_{\mathrm{pe}}(y, t)$ is the modified pulse-echo spatial impulse response that takes into consideration the geometry of the transducer to the spatial extent of the scattered field (beam diffraction). The pulse-echo impulse response, $h_{1}(t)$, for $\mathrm{CP}$ was generated by gating a sinusoid of 4 -cycles with a Hann window:

$$
w(n)= \begin{cases}0.5\left(1-\cos \left(\frac{2 \pi n}{L_{H}-1}\right)\right), & 0 \leq n \leq L_{H}-1, \\ 0, & \text { otherwise }\end{cases}
$$

where $n$ is an integer and $L_{H}$ is the number of samples in the window. The window and sinusoid parameters were chosen such that they match the transducer used in experiments. As a result, the pulse-echo impulse response generated was located at the focus of a 2.25-MHz single-element transducer $(f / 2.66)$ with a fractional bandwidth of $50 \%$ at $-3 \mathrm{~dB}$, which would correspond to a window length of $n=128$. For REC, the desired impulse response function, $h_{2}(t)$, was constructed to have double the fractional bandwidth or $100 \%$ at $-3 \mathrm{~dB}$, compared with CP method; therefore, a Hann window of size of half the length, $n=64$, was used. The spatial response for a circular focused piston source can be simulated as a circular Gaussian beam that is defined as

$$
h_{\mathrm{pe}}(y, t)=\delta\left(t-\frac{2 R_{d}}{c}\right) e^{-y^{2} / \sigma_{y}^{2}},
$$

where $R_{d}$ is the distance from the source to target in space, $c$ is the speed of sound of the medium, and $\sigma_{y}$, which is equal to $1.28 \mathrm{~mm}$, is the nominal lateral beamwidth of the source at $-6 \mathrm{~dB}$.

The received RF backscatter data were sampled at a rate of $100 \mathrm{MHz}$ and the transducer was translated laterally in increments of $0.1 \mathrm{~mm}$. The received RF data have a size of $4096 \times 58$ samples, axially and laterally. The object being imaged was a simulated phantom that was $20 \mathrm{~mm}$ long, $30 \mathrm{~mm}$ wide, and $1.92 \mathrm{~mm}$ high. A cylindrical target with a radius of $7.5 \mathrm{~mm}$ was located at the center of the phantom. To generate a hyperechoic target with a contrast of approximately $+6 \mathrm{~dB}$, the amplitude of the scatterers in the target lesion region was twice of the amplitude at the background. To achieve fully developed speckle, the phantom contained an average of 20 point scatterers per resolution cell volume. The scatterers were uniformly distributed throughout the phantom with random spatial locations. Thirty phantoms were simulated and evaluated with the image quality metrics discussed in Section 2.5. Attenuation and noise were not modeled in the simulations to examine the relationship of eREC-FC/despeckling filters to speckle effect only.

2.7. Experiments. Experiments were performed to validate the simulated results. A single-element weakly focused $(f / 2.66)$ transducer (Panametrics, Waltham, MA) with a center frequency of $2.25 \mathrm{MHz}$ was used to image a phantom by translating the transducer laterally. The transducer had a $-3-\mathrm{dB}$ bandwidth of $50 \%$ along with a pulse-echo beamwidth of $1.28 \mathrm{~mm}$. These parameters were measured using the wire technique [32] for transducer characterization. Using REC, the $-6-\mathrm{dB}$ pulse-echo bandwidth was 
enhanced to $100 \%$. There were two different experimental setups used: one for CP methods and another one for REC experiments. These setups would contain different noise levels due to the use of different excitation systems; therefore, to avoid errors in the comparisons, the noise levels were normalized to an eSNR of $28 \mathrm{~dB}$. Normalization of eSNR was accomplished by adding zero mean Gaussian white noise to the $\mathrm{CP}$ RF echo waveform after characterizing the eSNR from measurements of the signal with no scatterers. The two experimental setups are described as follows.

2.7.1. CP Experimental Setup. The transducer was excited by a pulser-receiver (5800, Panametrics, Waltham, MA) and the receive waveform was displayed on an oscilloscope (9354 TM, Lecroy, Chester Ridge, NY) for visual verification. The echo signal was recorded at a rate of $100 \mathrm{MHz}$ by a 12-bit A/D (Digitizing Board UF3025, Strategic Test, Woburn, MA) for further processing by a PC.

2.7.2. REC Experimental Setup. The preenhanced chirp was generated in MATLAB (MathWorks, Natick, MA) and downloaded to an arbitrary waveform generator (W1281A, Tabor Electronics, Tel Hanan, Israel). The excitation signal was sampled at a rate of $100 \mathrm{MHz}$ and amplified by an RF power amplifier (3251, ENI, Rochester, NY). The amplified signal $(50 \mathrm{~dB})$ was connected to the transducer through a diplexer (RDX-6, Ritec Enterprises, Warwick, RI). The echo signal was received by a pulser-receiver (5800, Panametrics, Waltham, MA), which was displayed on an oscilloscope (9354 TM, Lecroy, Chester Ridge, NY) for visual verification. The echo signal was recorded at a rate of $100 \mathrm{MHz}$ by a 12-bit A/D (Digitizing Board UF3025, Strategic Test, Woburn, MA) for further processing by a PC.

A tissue-mimicking phantom (Model 539, ATS Laboratories, Bridgeport, CT) was used to assess the performance of eREC-FC and the despeckling filters with the image quality metrics described in Section 2.5. The material from the tissue-mimicking phantom consisted of urethane rubber, which has a speed of sound of $1450 \mathrm{~m} / \mathrm{s} \pm 1.0 \%$ at $23^{\circ} \mathrm{C}$ and an attenuation coefficient of $0.5 \mathrm{~dB} / \mathrm{cm} / \mathrm{MHz} \pm 5.0 \%$. A $+6-\mathrm{dB}$ echogenic gray-scale target structure with a $15 \mathrm{~mm}$ diameter at a depth of $4 \mathrm{~cm}$ was imaged for both CP and REC. All measurements were conducted at room temperature in a tank of degassed water.

\section{Results and Discussion}

3.1. Computer Simulations. The CP reference, REC, REC-FC, and eREC-FC B-mode images along with the postprocessing despeckling filtered B-mode images are shown in Figure 4. The CNR, HO, and cSNR for the B-mode images are listed in Table 1. Histograms of the background and target regions for all of the images in Figure 4 are shown in Figure 5 while edge detection images are shown in Figure 6.

3.1.1. eREC-FC. Examination of the reference scans in Figures 4(a) and 4(b) revealed that by using the REC technique the speckle size was finer when compared with CP.
This finer speckle comes from the fact that the bandwidth was doubled, which translates into improvements in axial resolution. This smaller speckle size obtained by using REC is critical because the object boundaries are more defined compared with CP [18]. Application of frequency compounding to REC resulted in the B-mode image shown in Figure 4(c). In this scenario, subband widths that are 1/3 of the CP bandwidth were applied to the REC images. With REC-FC (third-width), significant improvements in visibility were observed but at the expense of blurring the image. Specifically, the CNR for REC-FC (third-width) resulted in an average improvement of $197 \%$ over 30 phantoms. CNR estimates are listed in Table 1. For eREC-FC, the CNR improved by an average of $148 \%$. However, in addition to the CNR enhancement, it was observed in the eREC-FC results shown in Figure 4(d) that the CNR enhancement was achieved while maintaining the axial resolution, as suggested in Figure 3, to comparable levels when exciting a transducer with a pulse. This result is significant as it suggests that improvements in CNR can be achieved without significantly degrading the axial resolution as shown in the REC-FC technique.

Histogram analysis was performed over the same regions used to obtain the estimates of CNR. The $\mathrm{HO}$ and $H_{\text {diff }}$ between the target region and the background regions are listed in Table 1. Previously, it was identified that using REC resulted in an image with a smaller speckle size. This improvement had no effect in minimizing the overlap between the target and background regions when compared to CP. However, by applying frequency compounding techniques such as REC-FC and eREC-FC, a substantial reduction in the $\mathrm{HO}$ was discovered. It should be noted that a $3.6 \%$ reduction in $\mathrm{HO}$ was observed in REC-FC (thirdwidth) over eREC-FC. Although eREC-FC has a slightly higher $\mathrm{HO}$, a reduction of $16.3 \%$ in $\mathrm{HO}$ was observed when compared to CP. Furthermore, REC-FC (third-width) did not provide any improvements in terms of the separation between the target and the background regions as measured in $H_{\text {diff }}$ compared to CP. On the contrary, eREC-FC provided a separation of 12 levels of pixel intensities to provide superior target detectability over REC-FC (third-width). Therefore, the slight increase in HO observed in eREC-FC compared to REC-FC (third-width) is acceptable given the benefits of improved spatial resolution and improved target detectability brought by using the eREC-FC technique.

As previously stated, REC-FC is known to enhance the boundaries of the lesions as shown in [18]. However, in eREC-FC, because images with variable speckle sizes are being compounded, it was observed that the transition between the target and the background was slightly blurred. Applying thresholding along with MS resulted in Figure 6. From the MS results, it was observed that REC-FC (thirdwidth) had a more pronounced boundary compared to eREC-FC. Consequently, the tradeoff in using eREC-FC is a degradation of the enhanced edges obtained with the RECFC technique in order to gain CNR while maintaining the same axial resolution as CP.

In terms of cSNR, REC-FC (third-width) provided the greatest amount of speckle reduction when compared to 


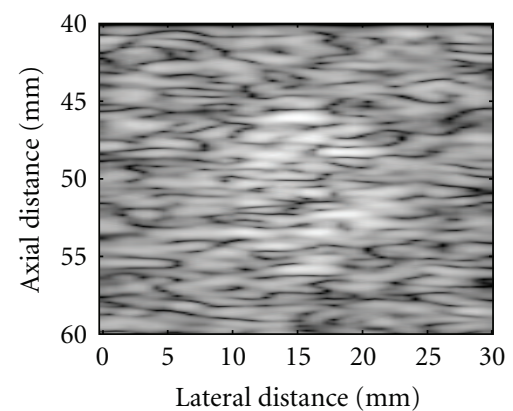

(a)

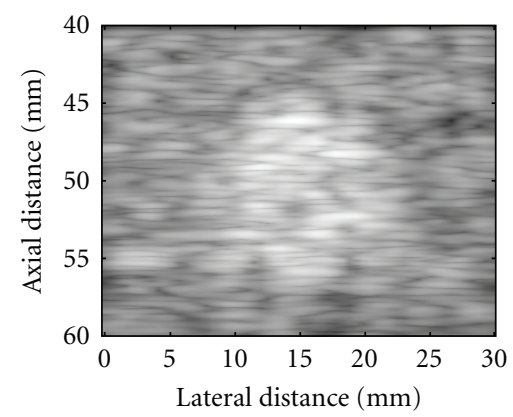

(d)

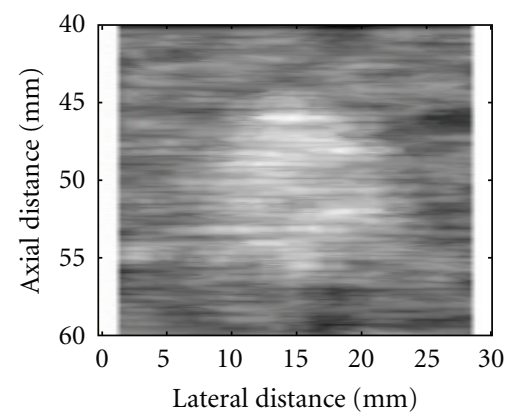

(g)

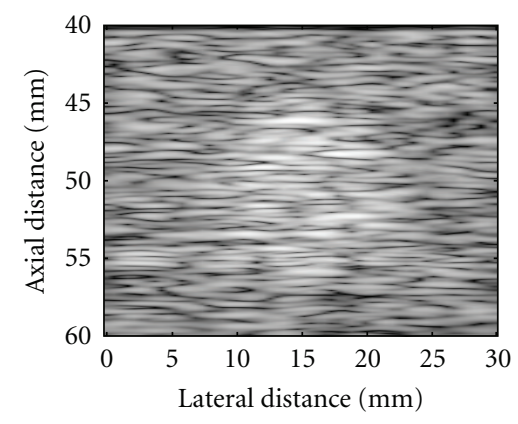

(b)

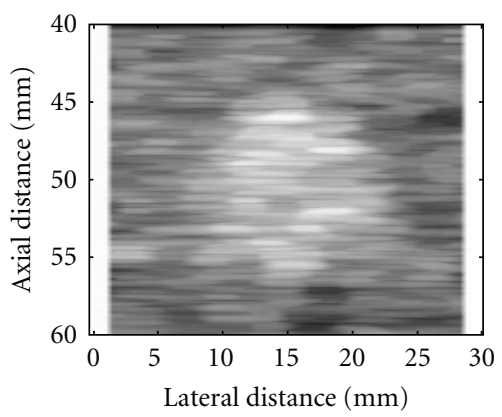

(e)

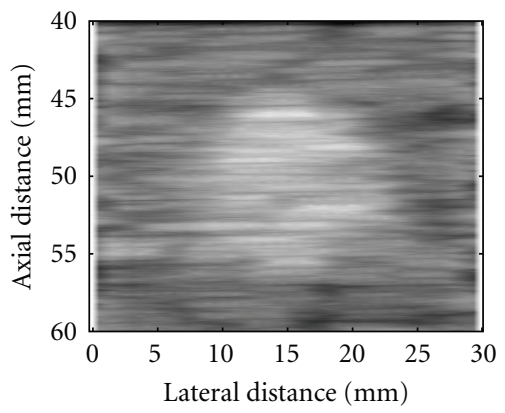

(h)

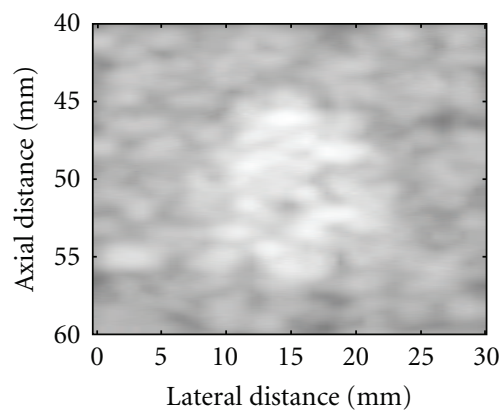

(c)

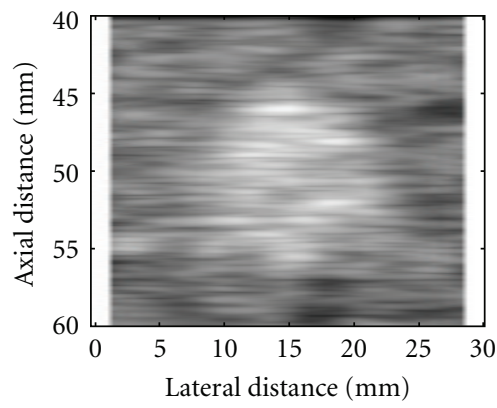

(f)

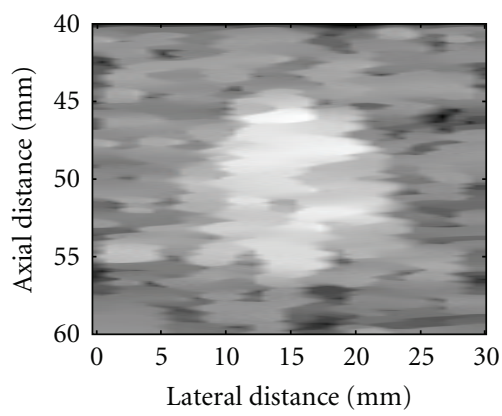

(i)

FIGURE 4: B-mode images of simulated results for the following: (a) CP and (b) REC reference scans, (c) REC-FC (third-width), (d) eRECFC, (e) eREC-FC with median filtering, (f) eREC-FC with Lee filtering, (g) eREC-FC with homogeneous mask area filtering, (h) eREC-FC with geometric filtering, and (i) eREC-FC with SRAD. Image dynamic range equals $-50 \mathrm{~dB}$.

TABLE 1: CNR, HO, $H_{\text {diff, }}$ and cSNR for the 30 cases of simulated RF data for a $15 \mathrm{~mm}$ target. ${ }^{1}$

\begin{tabular}{lcccc}
\hline Technique $^{2}$ & CNR & HO & $H_{\text {diff }}$ & cSNR \\
\hline CP & $0.728 \pm 0.172$ & $26.609 \pm 4.351$ & $36.358 \pm 8.265$ & - \\
REC & $0.730 \pm 0.146$ & $26.820 \pm 3.819$ & $35.920 \pm 6.809$ & $8.182 \pm 0.735$ \\
REC-FC (third-width) & $2.164 \pm 0.388$ & $6.699 \pm 3.258$ & $36.500 \pm 5.551$ & $14.200 \pm 0.564$ \\
eREC-FC & $1.806 \pm 0.301$ & $10.307 \pm 3.653$ & $48.590 \pm 7.496$ & $10.361 \pm 0.771$ \\
eREC-FC and median filtering & $2.192 \pm 0.405$ & $6.366 \pm 3.567$ & $58.334 \pm 8.923$ & $9.898 \pm 0.741$ \\
eREC-FC and Lee filtering & $2.296 \pm 0.401$ & $5.665 \pm 3.347$ & $57.477 \pm 8.563$ & $9.934 \pm 0.724$ \\
eREC-FC and HMA filtering & $2.214 \pm 0.352$ & $6.335 \pm 3.073$ & $56.950 \pm 8.070$ & $9.873 \pm 0.712$ \\
eREC-FC and geometric filtering & $2.154 \pm 0.363$ & $6.962 \pm 3.099$ & $50.248 \pm 7.148$ & $11.027 \pm 0.647$ \\
eREC-FC and SRAD filtering & $2.328 \pm 0.454$ & $5.167 \pm 3.170$ & $58.866 \pm 9.649$ & $17.338 \pm 0.480$ \\
$P$ value & $2.252 \times 10^{-77}$ & $3.900 \times 10^{-105}$ & $2.482 \times 10^{-047}$ & $1.922 \times 10^{-142}$ \\
\hline
\end{tabular}

${ }^{1}$ The values in the table are described in terms of the mean plus/minus one standard deviation.

${ }^{2} \mathrm{CP}$ : conventional pulsing; REC: resolution enhancement compression; FC: frequency compounding; eREC-FC: enhanced REC-FC; HMA: homogeneous mask area filtering; SRAD: speckle-reducing anisotropic diffusion. 


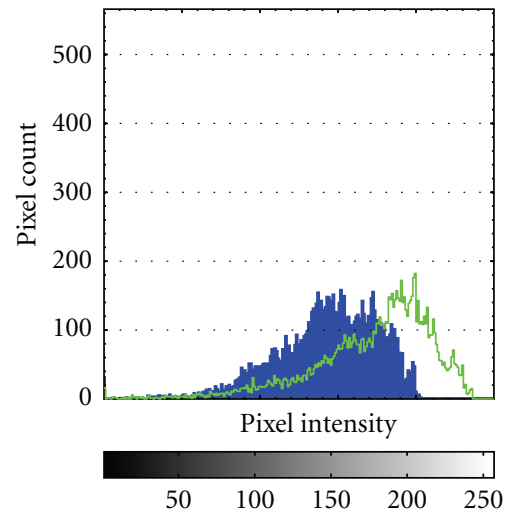

(a)

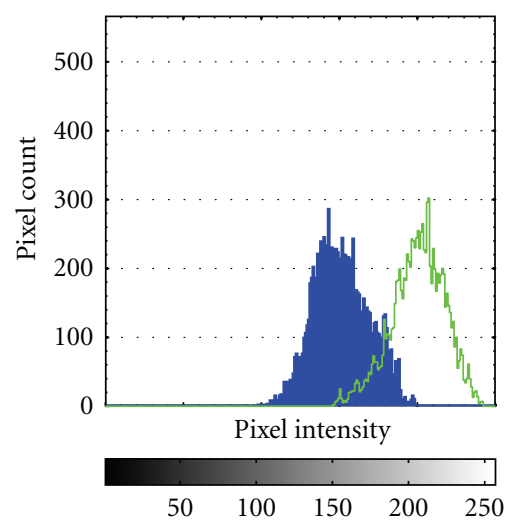

(d)

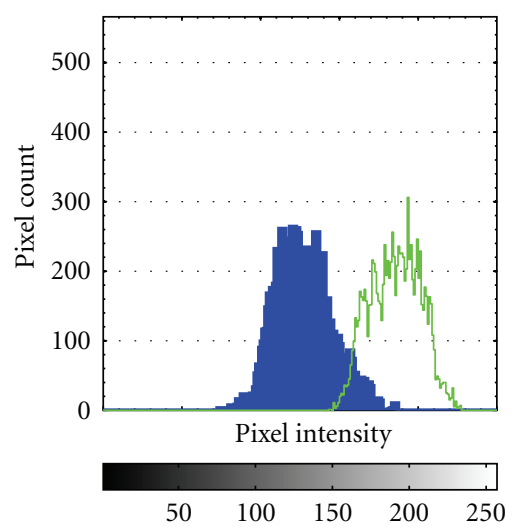

(g)

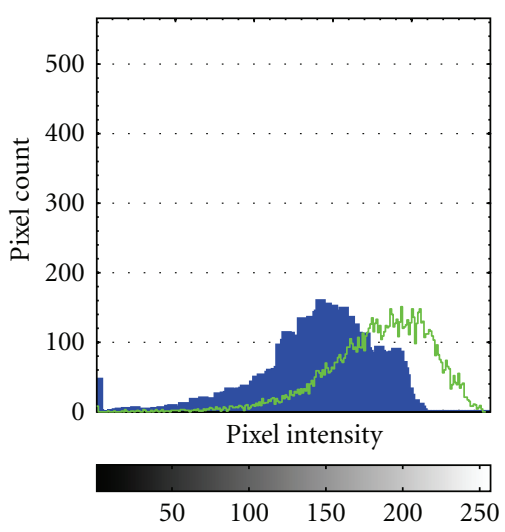

(b)

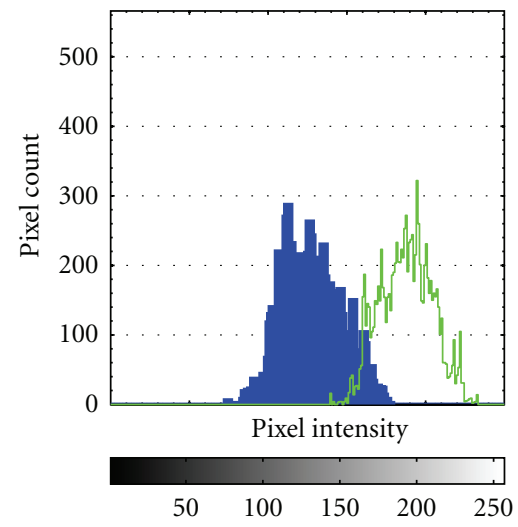

(e)

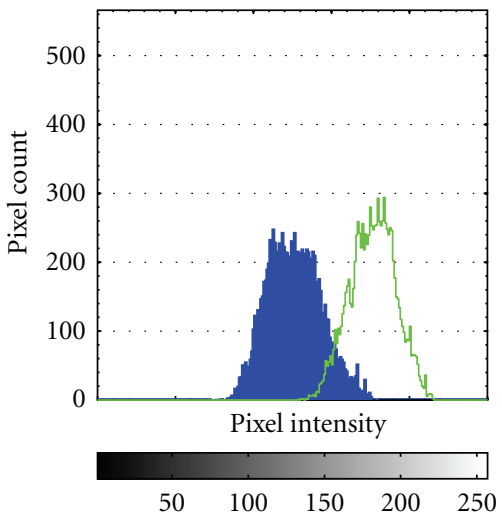

(h)

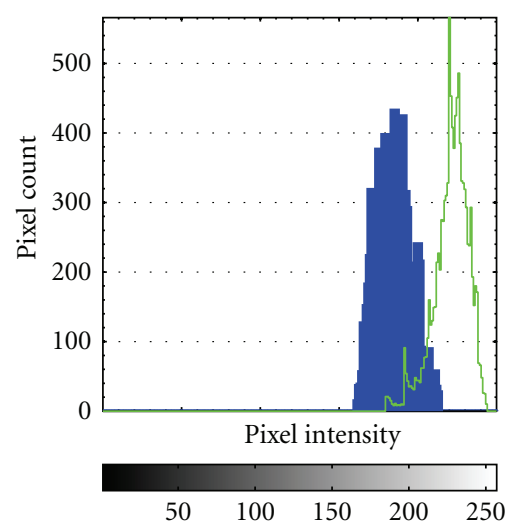

(c)

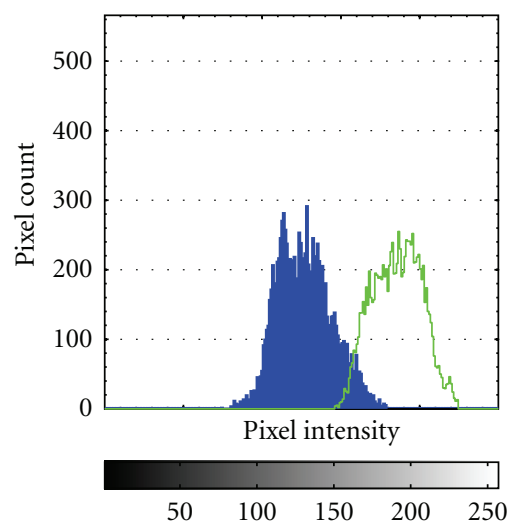

(f)

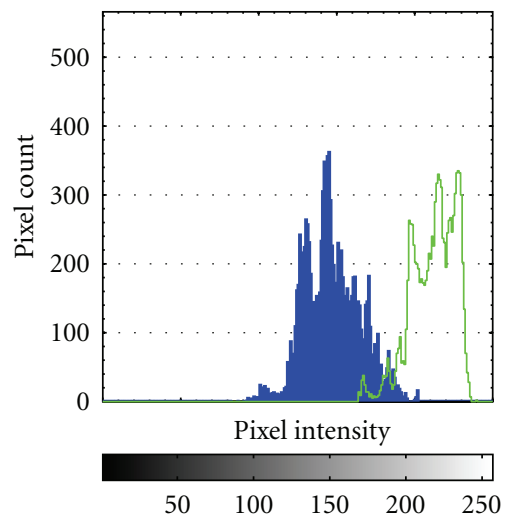

(i)

FIGURE 5: Histograms of simulated results for the following: (a) CP and (b) REC reference scans, (c) REC-FC (third-width), (d) eREC-FC, (e) eREC-FC with median filtering, (f) eREC-FC with Lee filtering, (g) eREC-FC with homogeneous mask area filtering, (h) eREC-FC with geometric filtering, and (i) eREC-FC with SRAD (dark: background region; light: target region).

the reference CP image. However, REC-FC (third-width) suffers from a degradation in axial resolution as the subband widths were $1 / 3$ of the original CP bandwidth. Conversely, eREC-FC provided some reduction in speckle without the deterioration in axial resolution obtained in REC-FC (thirdwidth). Consequently, because eREC-FC provided CNR improvements while maintaining spatial resolution along with improvements as indicated by the comparative metrics, eREC-FC was further evaluated by applying postprocessing despeckling filters.
3.1.2. Postprocessing Speckle Reduction Techniques. In this section, the images generated using the eREC-FC technique were modified by applying several postprocessing despeckling filters discussed in Section 2.4. For this study, the size of the filtering window for the median, Lee, and the homogeneous mask area techniques was $7 \times 7$. The units of the pixels are one beamwidth by one time sample. For the geometric and SRAD techniques, 5 and 3300 iterations were applied, respectively. In this study, the main focus was to quantify the improvements provided by each technique using the image 


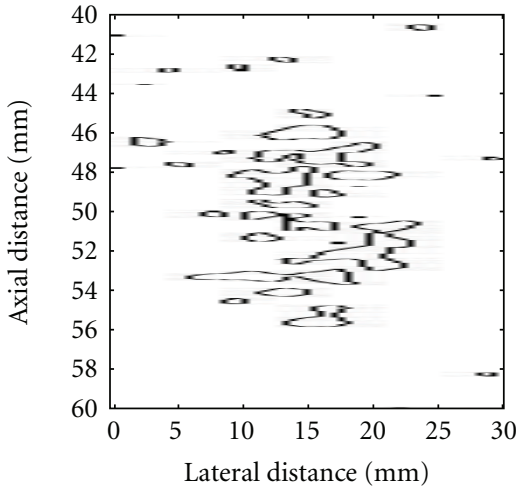

(a)

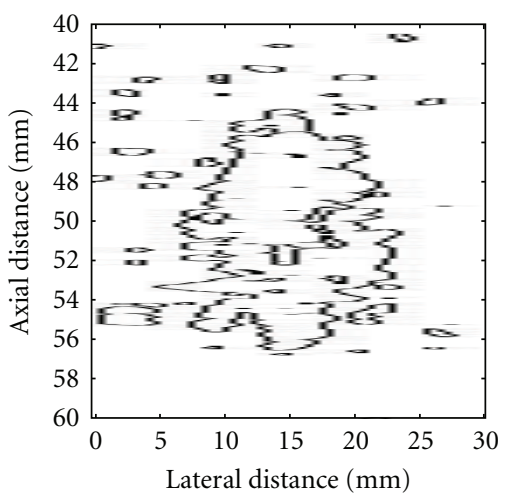

(d)

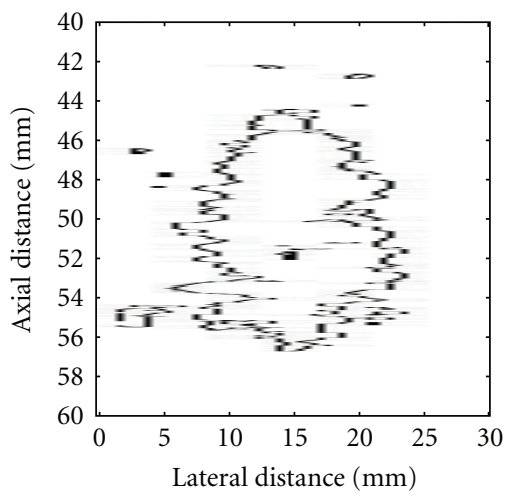

(g)

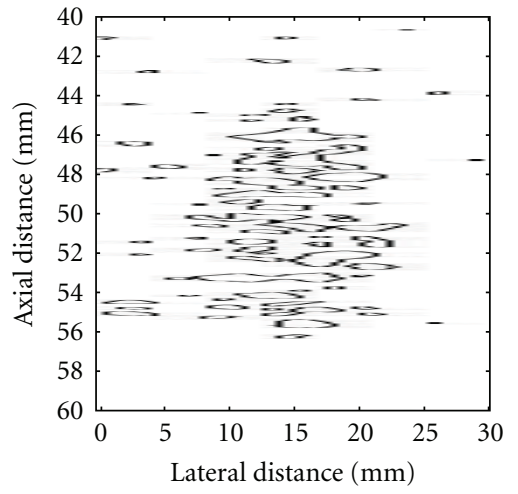

(b)

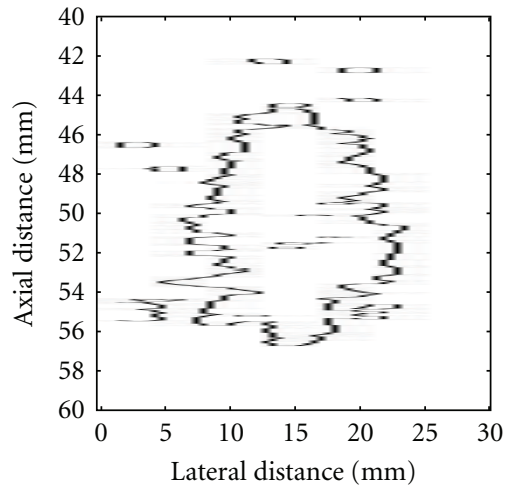

(e)

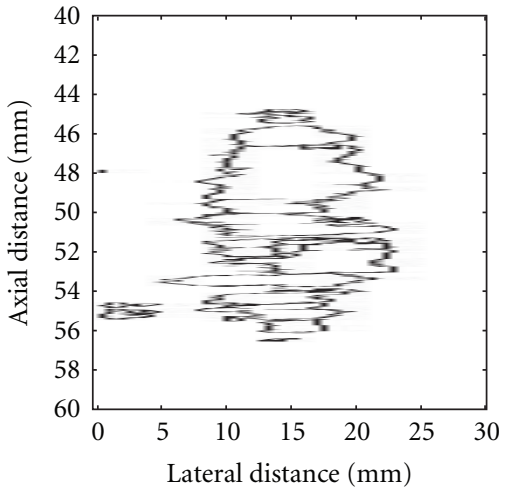

(h)

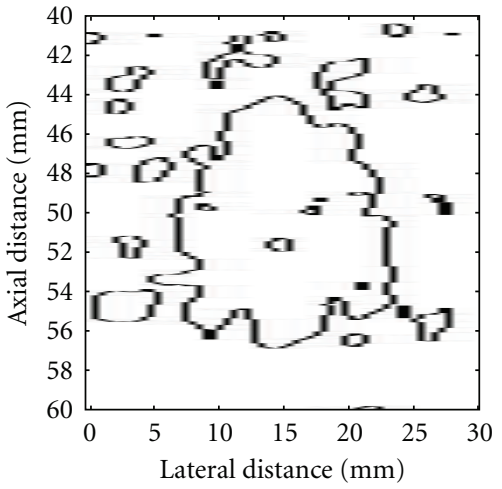

(c)

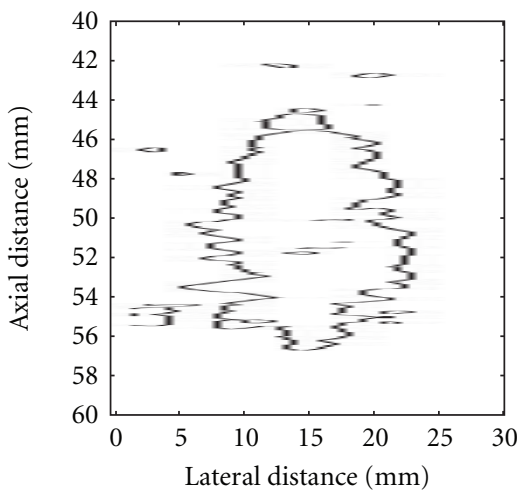

(f)

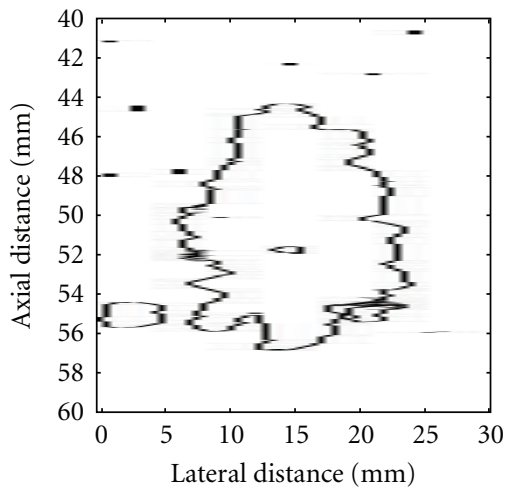

(i)

FIGURE 6: Edge detection images of simulated results for the following: (a) CP and (b) REC reference scans, (c) REC-FC (third-width), (d) eREC-FC, (e) eREC-FC with median filtering, (f) eREC-FC with Lee filtering, (g) eREC-FC with homogeneous mask area filtering, (h) eREC-FC with geometric filtering, and (i) eREC-FC with SRAD.

quality metrics discussed in Section 2.5. Filter computational requirements for all the filters studied herein are evaluated in [33]. Moreover, several real-time implementations of the iterative SRAD technique are evalulated in [34].

Examination of the filtered images in Figures 4(e) and 4(i) revealed that CNR improvements were obtained when using postprocessing despeckling filters compared to $\mathrm{CP}$ and eREC-FC. When compared to REC-FC (third-width) all cases resulted in improvements except in the case where the geometric filter was applied. However, the difference in CNR between REC-FC (third-width) and eREC-FC with geometric filtering was almost negligible (0.01). For the eREC-FC image with a median filter shown in Figure 4(e) it was observed that a smearing of the pixels with similar intensities in the lateral extent occurred. A similar smearing was observed in Figure 4(f), which shows the eREC-FC image with a Lee filter. However, the smearing is more prominent across the target and background boundary. For the eREC-FC image with homogeneous mask area filtering, shown in Figure 4(g), a noisy pattern appears around the boundary between the target and the background. In Figure $4(\mathrm{~h})$, the eREC-FC image with a geometric filter is 


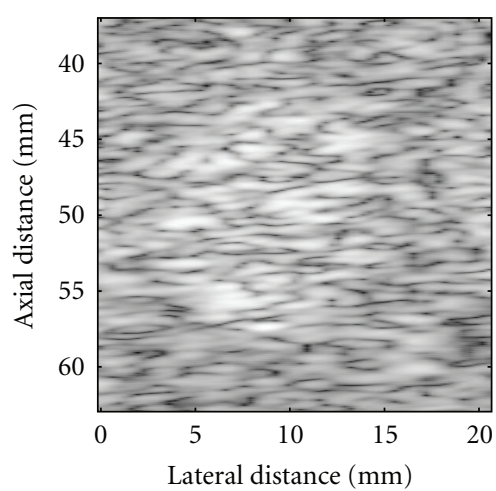

(a)

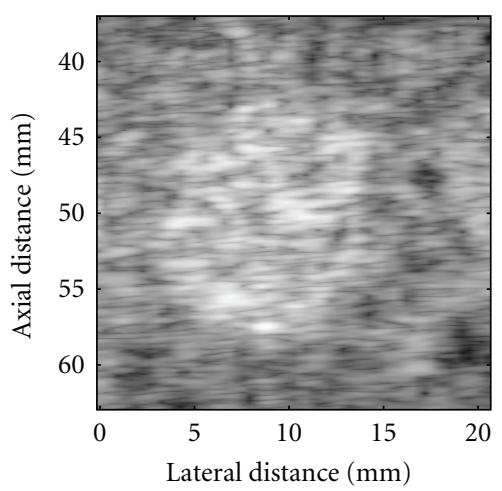

(d)

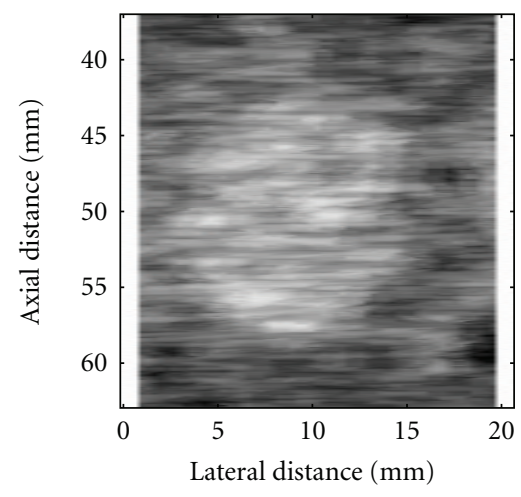

(g)

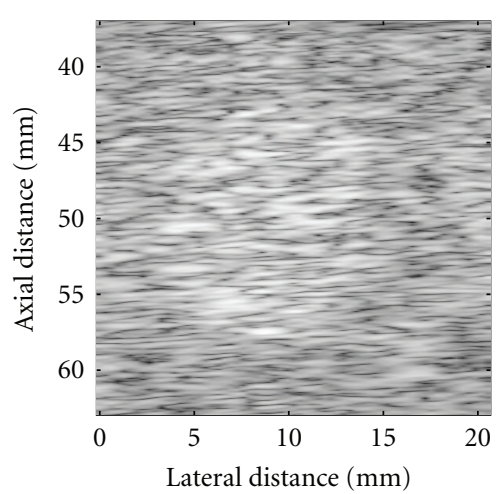

(b)

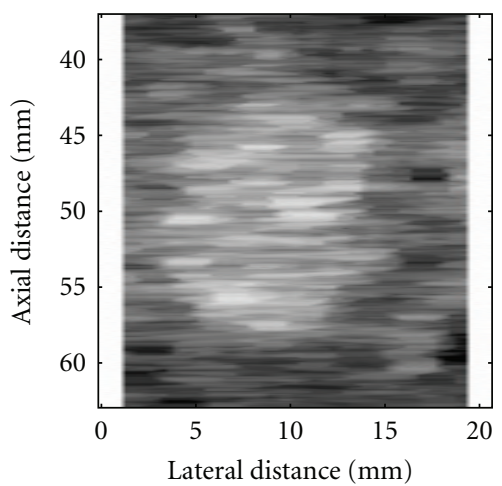

(e)

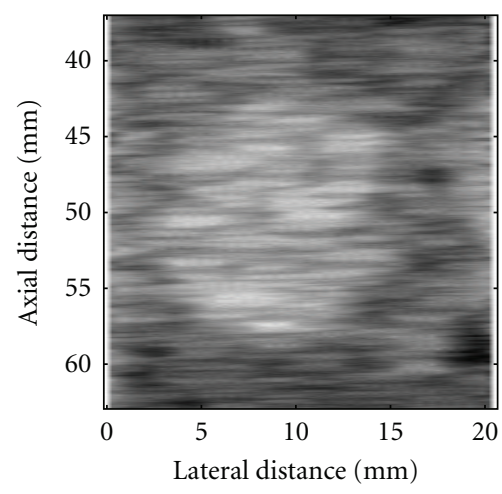

(h)

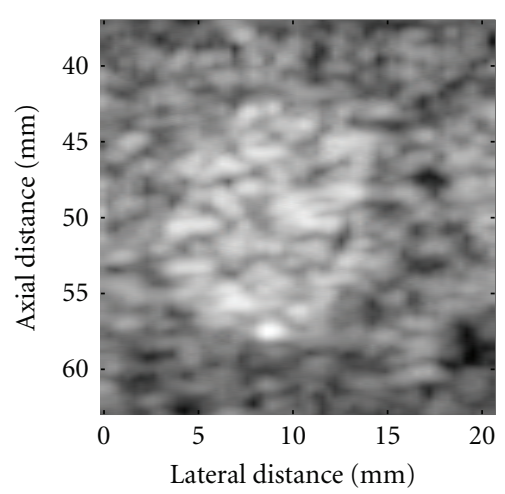

(c)

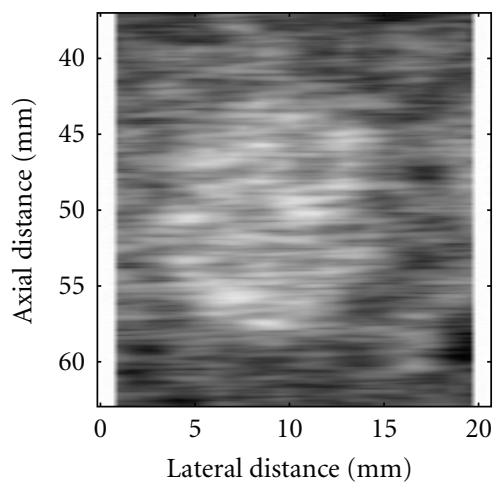

(f)

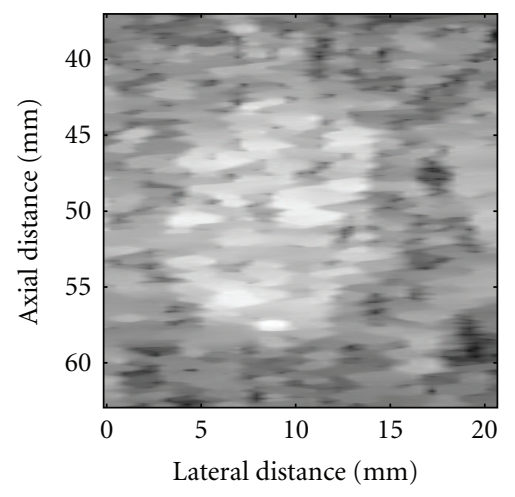

(i)

FIGURE 7: B-mode images of experimental measurement for the following: (a) CP and (b) REC reference scans, (c) REC-FC (third-width), (d) eREC-FC, (e) eREC-FC with median filtering, (f) eREC-FC with Lee filtering, (g) eREC-FC with homogeneous mask area filtering, (h) eREC-FC with geometric filtering, and (i) eREC-FC with SRAD. Image dynamic range equals $-50 \mathrm{~dB}$.

shown. For the geometric filtering case a similar appearance to Lee filter was observed. Finally, it was observed that for eREC-FC with SRAD, shown in Figure 4(i), the speckle was replaced by a blotchy appearance that was able to enhance or clearly demarcate the edges in the image. The CNR for eRECFC in conjunction with postprocessing despeckling filters is listed in Table 1. The highest CNR was achieved when applying SRAD to the eREC-FC images. Overall, by using despeckling filters in conjunction with eREC-FC the levels of CNR estimated for REC-FC (third-width) were exceeded.

Histogram analysis was performed over the same regions used to obtain the estimates of CNR. The $\mathrm{HO}$ and $H_{\text {diff }}$ between the target region and the background regions are listed in Table 1. Application of postprocessing despeckling filters to the eREC-FC images resulted in decreases in the range from 3.35 to 5.14 for HO. In addition, improvements in terms of the separation between the target and the background mean pixel intensities in the range of 1.57 to 10.27 were observed. This separation improves the overall target detectability. In Section 3.1.1 it was identified that the HO was the smallest for REC-FC (third-width) and the biggest separation between the target and background mean pixel intensities was for eREC-FC. By using despeckling filters, the HO was reduced beyond REC-FC (third-width) 


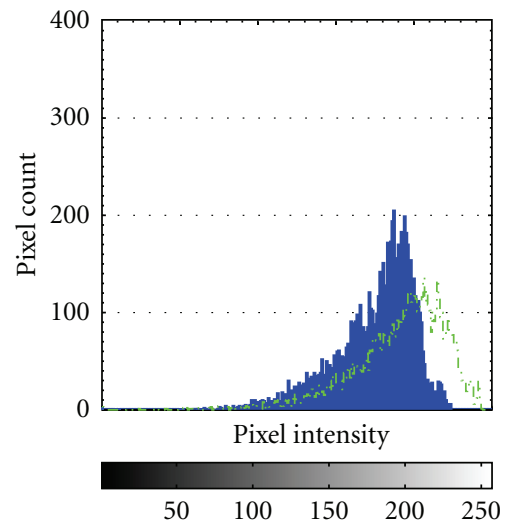

(a)

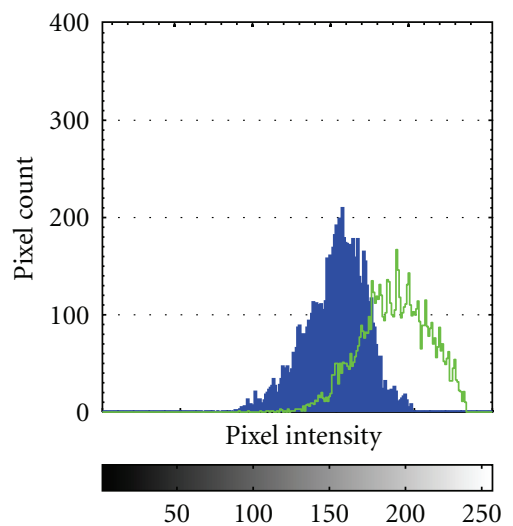

(d)

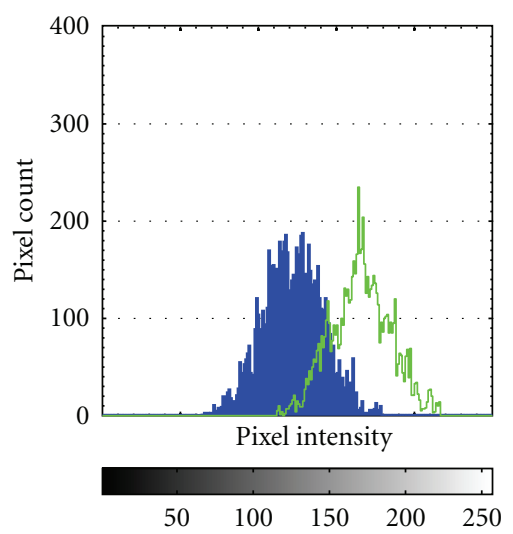

(g)

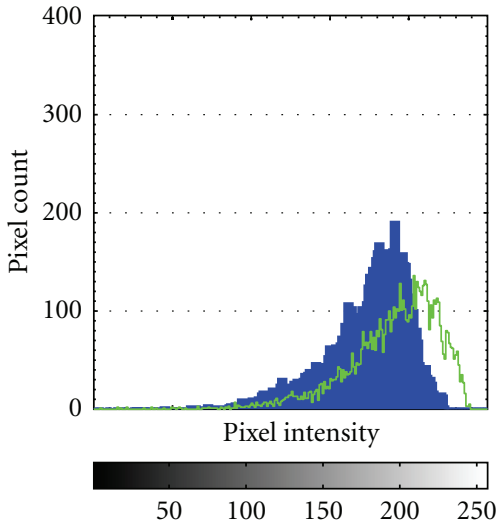

(b)

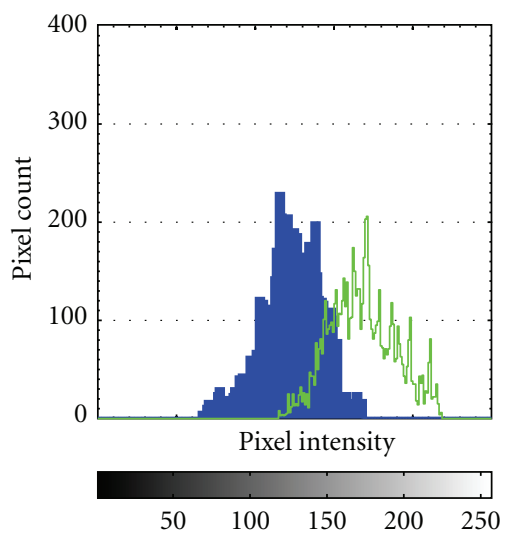

(e)

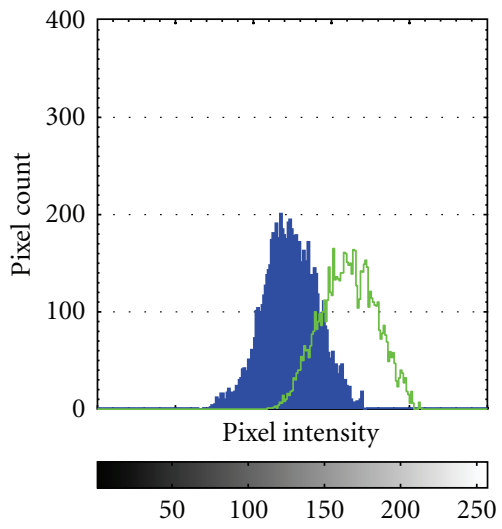

(h)

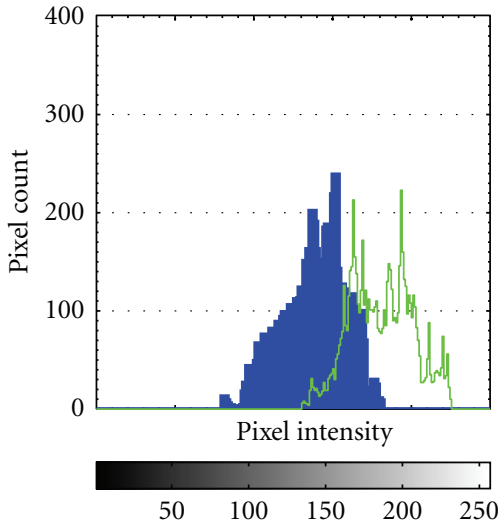

(c)

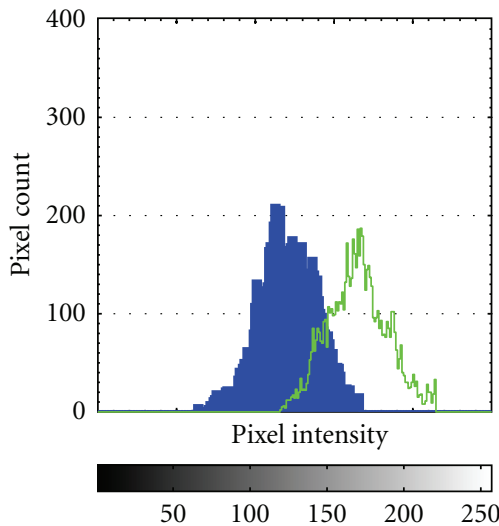

(f)

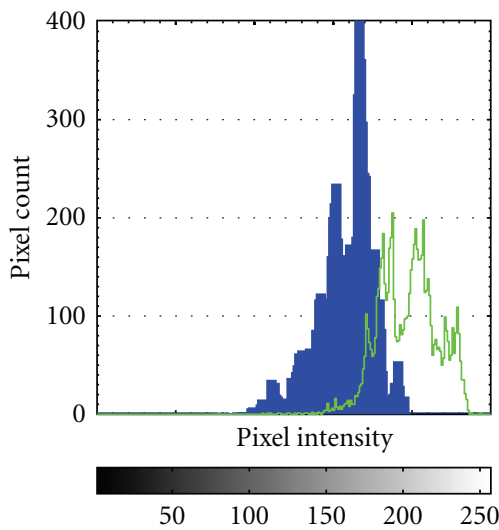

(i)

Figure 8: Histograms of experimental measurements for the following: (a) CP and (b) REC reference scans, (c) REC-FC (third-width), (d) eREC-FC, (e) eREC-FC with median filtering, (f) eREC-FC with Lee filtering, (g) eREC-FC with homogeneous mask area filtering, (h) eREC-FC with geometric filtering, and (i) eREC-FC with SRAD (dark: background region; light: target region).

levels while separating the target and background mean pixel intensities beyond the levels for eREC-FC. Consequently, improved CNR and target detectability was achieved with all despeckling filters.

Application of thresholding along with MS to the eRECFC images that were processed with despeckling filters resulted in Figures 6(e) and 6(i). From the MS results, it was observed that the median, Lee, homogeneous mask area, and SRAD produced improved target delineation when compared to eREC-FC. eREC-FC with geometric filtering showed some horizontal striations that masked the outline of the target. Furthermore, it was noted that SRAD had a similar outline as REC-FC (third-width). Recall that with eREC-FC a tradeoff of degradation in edges versus CNR enhancement while maintaining the same axial resolution as $\mathrm{CP}$ was observed. Application of despeckling filters, except for the geometric filtering case, extended this tradeoff. Consequently, postprocessing despeckling filters in conjunction 


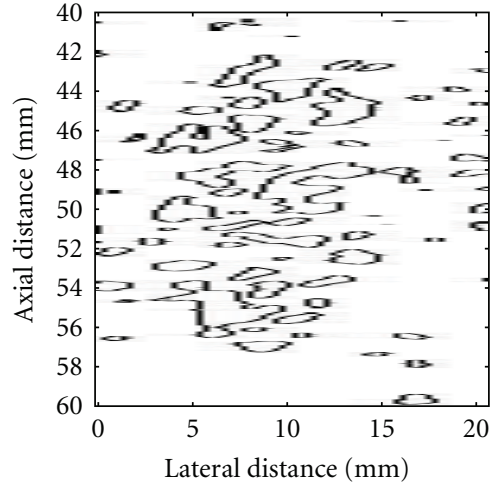

(a)

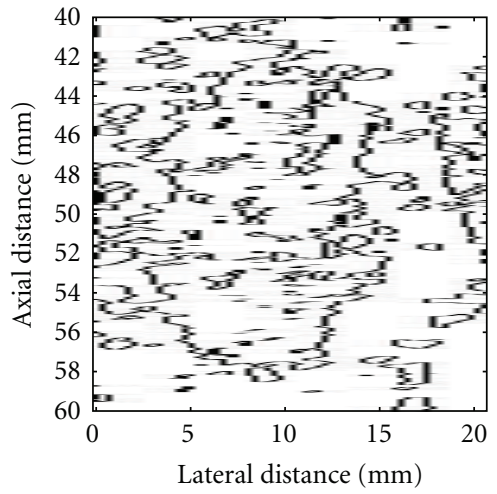

(d)

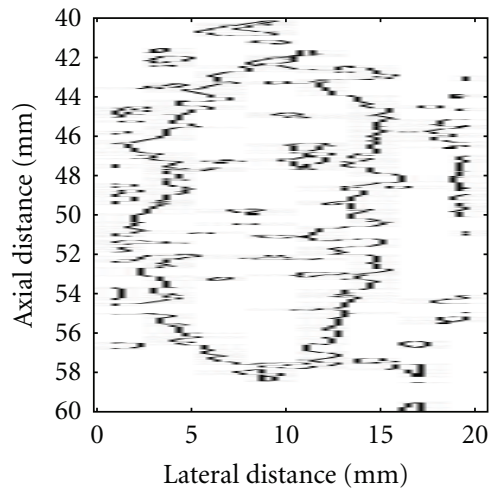

(g)

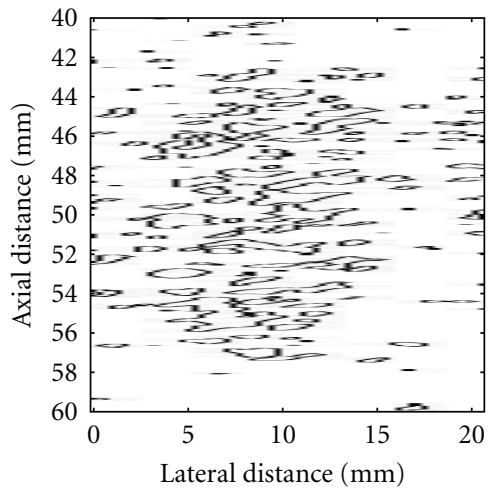

(b)

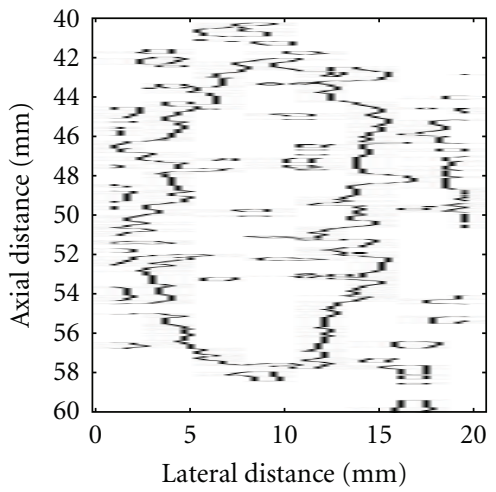

(e)

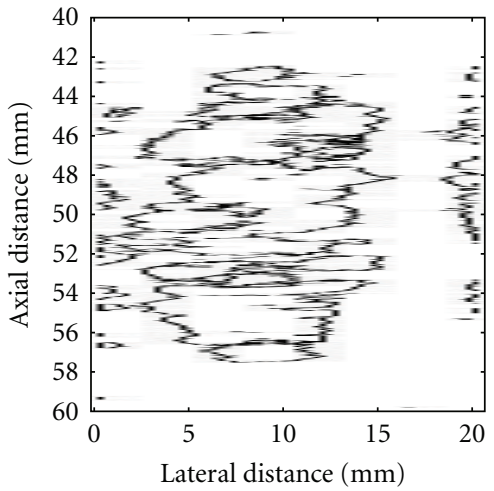

(h)

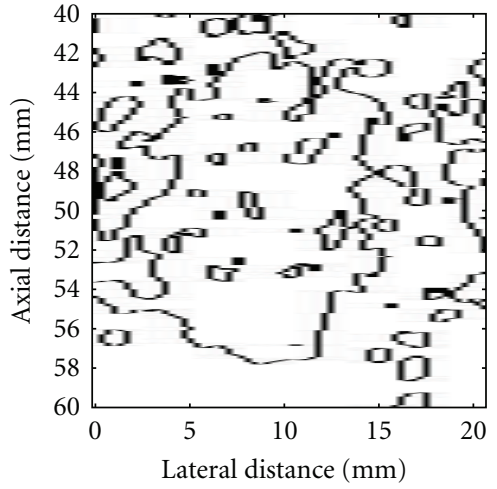

(c)

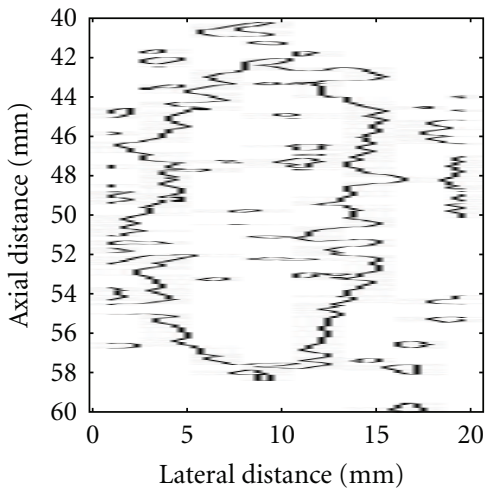

(f)

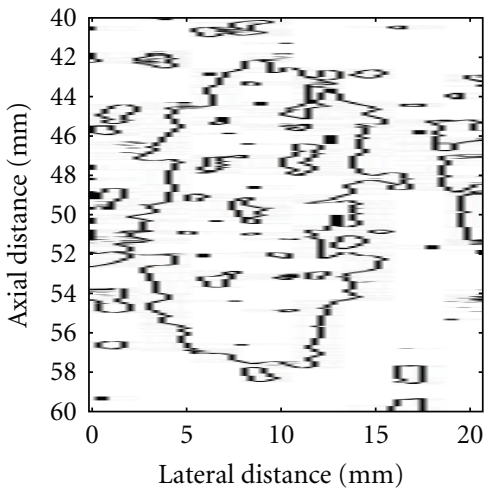

(i)

FIGURE 9: Edge detection images of experimental measurements for the following: (a) CP and (b) REC reference scans, (c) REC-FC (thirdwidth), (d) eREC-FC, (e) eREC-FC with median filtering, (f) eREC-FC with Lee filtering, (g) eREC-FC with homogeneous mask area filtering, (h) eREC-FC with geometric filtering, and (i) eREC-FC with SRAD.

with eREC-FC improved the overall target outline while improving target detectability.

Evaluation of the comparative metric indicates that the performance of the various despeckling filters varies. For example, there was observed a reduction in the cSNR for eREC-FC with median, Lee, and homogeneous mask area filtering while an improvement in cSNR was achieved for the geometric and SRAD filtering. The implication is that only the geometric and SRAD filtering reduced the speckle beyond the eREC-FC image. Although reduced cSNR was observed for the median, Lee, and homogeneous mask area filtering techniques, the reductions were small. Overall, eREC-FC combined with SRAD resulted in the best cSNR when compared to the other despeckling filters.

For the simulations, the aforementioned results suggest that eREC-FC is a useful technique to enhance target detectability while improving image CNR and maintaining a spatial resolution comparable to CP. The performance of eREC-FC was further improved by applying postprocessing despeckling filters. In summary, eREC-FC combined with 
TABLE 2: CNR, HO, $H_{\text {diff, }}$ and cSNR for the $15 \mathrm{~mm}$ ATS phantom target.

\begin{tabular}{|c|c|c|c|c|}
\hline Technique $^{1}$ & CNR & $\mathrm{HO}$ & $H_{\text {diff }}$ & cSNR \\
\hline $\mathrm{CP}$ & 0.56 & 32.49 & 16.06 & - \\
\hline REC & 0.62 & 32.49 & 17.61 & 8.23 \\
\hline REC-FC (third-width) & 1.73 & 14.07 & 43.17 & 12.82 \\
\hline eREC-FC & 1.60 & 17.22 & 37.64 & 8.72 \\
\hline eREC-FC and median filtering & 2.29 & 12.12 & 45.43 & 8.08 \\
\hline eREC-FC and Lee filtering & 2.57 & 12.09 & 44.89 & 8.14 \\
\hline eREC-FC and HMA filtering & 2.51 & 13.41 & 43.98 & 8.12 \\
\hline eREC-FC and geometric filtering & 1.83 & 15.17 & 37.67 & 10.07 \\
\hline eREC-FC and SRAD filtering & 1.86 & 10.65 & 40.49 & 18.32 \\
\hline
\end{tabular}

${ }^{1} \mathrm{CP}$ : conventional pulsing; REC: resolution enhancement compression; FC: frequency compounding; eREC-FC: enhanced REC-FC; HMA: homogeneous mask area filtering; SRAD: speckle reducing anisotropic diffusion.

SRAD, as quantified by the metrics discussed in Section 2.5, emerged as the best technique that significantly improves the quality of ultrasonic images.

3.2. Experiments. The CP reference, REC, REC-FC, and eREC-FC B-mode images along with the postprocessing despeckling filtered B-mode images are shown in Figure 7. The CNR, HO, and cSNR for the B-mode images are listed in Table 2. Histograms of the background and target regions for all of the images in Figure 7 are shown in Figure 8 while edge detection images are shown in Figure 9.

3.2.1. eREC-FC. Similar to simulations, eREC-FC resulted in $\mathrm{CNR}, \mathrm{HO}$, and $H_{\text {diff }}$ improvements when compared to $\mathrm{CP}$ without significantly degrading the axial resolution. A significant deviation from the simulations was observed when evaluating the $H_{\text {diff }}$ for REC-FC (third-width). Both schemes, REC-FC (third width) and eREC-FC, improved target detectability by separating the mean of the target and background regions. In simulations, only deviations were observed for eREC-FC. Evaluating the histogram data listed in Table 2 suggests that the best target detectability was obtained with REC-FC (third-width) because of the combination of a smaller $\mathrm{HO}$ and a greater separation between the target and background mean intensity. However, the difference between eREC-FC and REC-FC (third-width) were minimal compared to the improvement both techniques obtained compared to CP. Therefore, by averaging the CNR of all the REC-FC cases used to generate the eREC-FC image also resulted in a CNR value in between the half width and third-width REC-FC cases. This would suggest that an approximation of the CNR improvements obtained with eREC-FC can be established by averaging the CNR of the images being compounded. Furthermore, the CNR improvements obtained with eREC-FC were achieved without deteriorating the axial resolution beyond CP levels, which is the main detriment of the REC-FC technique.

3.2.2. Postprocessing Speckle Reduction Techniques. In this section, the images generated from experimental measurements using the eREC-FC technique were modified by applying several postprocessing despeckling filters discussed in Section 2.4.

Examination of the filtered images in Figures 7(e)7(i) revealed that CNR improvements were obtained when using postprocessing despeckling filters compared to $\mathrm{CP}$ and eREC-FC. Unlike simulations, all cases resulted in improvements when compared to REC-FC (third-width). The CNR for eREC-FC in conjunction with postprocessing despeckling filters is listed in Table 2. The highest CNR was achieved when applying the Lee filter to the eREC-FC technique. In terms of CNR, the Lee filter in conjunction with the eREC-FC technique was the second best technique as determined by the simulations. Moreover, it was determined in the simulations that eREC-FC in conjunction with SRAD provided the best visibility. However, this was not true in the experiments although the relative improvements for simulations and experiment were quite similar when using SRAD. The significant difference between the eRECFC images for the simulation and experiment is that a larger overlap in pixel intensity between the background and the target occurs during the experiment. Consequently, the experimental measurements allow for improvements without saturating the effectiveness of the despeckling filters, which could have occurred during the simulations. Moreover, unlike simulations, all of the despeckling filters when combined with eREC-FC improved the image CNR beyond what was obtained when using REC-FC (thirdwidth). In fact, in simulations the largest improvement over REC-FC (third-width) was approximately 7\% when combining eREC-FC with SRAD, while in the experiments an improvement of $49 \%$ was achieved over REC-FC (thirdwidth) when combining eREC-FC with the Lee filter. Overall, by using despeckling filtering in conjunction with eREC-FC significant improvements in CNR were obtained over RECFC (third-width) along with improvements in terms of the spatial resolution because the eREC-FC image was used as the reference filtered image.

For histogram analysis and cSNR, similar trends were observed in the experimental measurements as predicted by the computer simulations. As in simulations, all postprocessing despeckling filters reduced the HO below REC-FC (thirdwidth) levels except the geometric filtering case. However, 
in the experimental measurements, $H_{\text {diff }}$ for the geometric filtering case resulted in a smaller separation between the target and background histograms when compared to the REC-FC (third-width) case.

The aforementioned experimental results validate the simulation findings listed in Section 3.1. Overall, the results suggest that eREC-FC is a useful technique to enhance target detectability while improving image CNR and maintaining a spatial resolution comparable to CP. Also, the performance of eREC-FC was further improved by applying postprocessing despeckling filters. In summary, eREC-FC combined with Lee provided the best improvement in terms of CNR while SRAD provided the best improvement in terms of target detectability and speckle reduction. Therefore, both of these techniques significantly improved the quality of ultrasonic images beyond what is available when using CP, REC, RECFC (third-width), and eREC-FC.

\section{Conclusions}

A technique that improves target visibility in ultrasound images, known as eREC-FC, was proposed. It was observed that with eREC-FC the quality of the B-mode images generated from echoes of simulated and experimental tissuemimicking phantoms was drastically improved by increasing the CNR. The CNR values obtained with eREC-FC were observed to be within the CNR values estimated for the halfwidth and third-width REC-FC cases that were determined in a previous study [18]. A potential detriment to eREC-FC technique would be if the difference in contrast between the background and the target is larger than $20 \mathrm{~dB}$. As shown in Figure 3 the axial resolution at $-20 \mathrm{~dB}$ is double of that for CP. Therefore, a smearing in the image, similar to that observed in the REC-FC study, is possible under targets with large contrast difference with the background (i.e., cystic targets). A potential solution would be to evaluate the image using a sliding window by applying spatial filter that preserves brightness at the edges (i.e., keep the original pixel in the image) and smooths the original image otherwise (i.e., replace original pixel in the image by the pixel obtained with eREC-FC technique). The potential tradeoff with this solution could be that small targets, depending on the size of the sliding window, may not be improved using a spatial eREC-FC technique.

By itself, the eREC-FC provided substantial improvements in image visibility compared to CP and REC. However, the REC-FC (third-width) appeared to provide better image visibility compared to eREC-FC. Although eREC-FC improved the CNR of ultrasonic B-mode images, further improvements were obtained by applying several postprocessing despeckling filter schemes. These techniques include median filtering, Lee filtering, homogeneous mask area filtering, geometric filtering, and speckle-reducing anisotropic diffusion. Simulations and experimental measurements were used to establish the usefulness of the combination of the eREC-FC technique with despeckling filters in enhancing image CNR, improving target detectability, and reducing speckle noise. Simulations and experimental measurements suggest that eREC-FC combined with despeckling filters was a useful tool to obtain substantial improvements in terms of image visibility and to enhance the boundaries between the target and the background.

\section{References}

[1] C. B. Burckhardt, "Speckle in ultrasound B-mode scans," IEEE Transactions on Sonics and Ultrasonics, vol. 25, no. 1, pp. 1-6, 1978.

[2] M. S. Patterson and F. S. Foster, "The improvement and quantitative assessment of B-mode images produced by an annular array/cone hybrid," Ultrasonic Imaging, vol. 5, no. 3, pp. 195213, 1983.

[3] S. K. Jespersen, J. E. Wilhjelm, and H. Sillesen, "Multi-angle compound imaging," Ultrasonic Imaging, vol. 20, no. 2, pp. 81-102, 1998.

[4] G. E. Trahey, S. W. Smith, and O. T. von Ramm, "Speckle pattern correlation with lateral aperture translation: experimental results and implications for spatial compounding," Modelling, Measurement and Control A, vol. 33, no. 3, pp. 257-264, 1986.

[5] A. R. Groves and R. N. Rohling, "Two-dimensional spatial compounding with warping," Ultrasound in Medicine and Biology, vol. 30, no. 7, pp. 929-942, 2004.

[6] P. Soler, C. Delso, N. Villain, E. Angelini, and I. Bloch, "Superresolution spatial compounding techniques, with application to $3 \mathrm{D}$ breast ultrasound imaging," in Medical Imaging 2006: Ultrasonic Imaging and Signal Processing, vol. 6147 of Proceedings of SPIE, pp. 281-292, San Diego, Calif, USA, February 2006.

[7] J. G. Abbott and F. L. Thurstone, "Acoustic speckle: Theory and experimental analysis," Ultrasonic Imaging, vol. 1, no. 4, pp. 303-324, 1979.

[8] S. M. Gehlbach and F. G. Sommer, "Frequency diversity speckle processing," Ultrasonic Imaging, vol. 9, no. 2, pp. 92105, 1987.

[9] P. A. Magnin, O. T. von Ramm, and F. L. Thurstone, "Frequency compounding for speckle contrast reduction in phased array images," Ultrasonic Imaging, vol. 4, no. 3, pp. 267-281, 1982.

[10] H. E. Melton and P. A. Magnin, "A-mode speckle reduction with compound frequencies and compound bandwidths," Ultrasonic Imaging, vol. 6, no. 2, pp. 159-173, 1984.

[11] V. L. Newhouse, N. M. Bilgutay, J. Saniie, and E. S. Furgason, "Flaw-to-grain echo enhancement by split-spectrum processing," Ultrasonics, vol. 20, no. 2, pp. 59-68, 1982.

[12] T. S. Huang, G. J. Yang, and G. Y. Tang, "A fast twodimensional median filtering algorithm," IEEE Transactions on Acoustics, Speech and Signal Processing, vol. 27, no. 1, pp. 13$18,1979$.

[13] M. O. Ahmad and D. Sundararajan, "A fast algorithm for twodimensional median filtering," IEEE Transactions on Circuits and Systems, vol. 34, no. 11, pp. 1364-1374, 1988.

[14] J. S. Lee, "Digital image enhancement and noise filtering by using local statistics," IEEE Transactions on Pattern Analysis and Machine Intelligence, vol. 2, no. 2, pp. 165-168, 1980.

[15] D. T. Kuan, A. A. Sawchuk, T. C. Strand, and P. Chavel, "Adaptive restoration of images with speckle," IEEE Transactions on Acoustics, Speech, and Signal Processing, vol. 35, no. 3, pp. 373383, 1987.

[16] Y. Yu and S. T. Acton, "Speckle reducing anisotropic diffusion," IEEE Transactions on Image Processing, vol. 11, no. 11, pp. 1260-1270, 2002. 
[17] K. Z. Abd-Elmoniem, A. B. M. Youssef, and Y. M. Kadah, "Real-time speckle reduction and coherence enhancement in ultrasound imaging via nonlinear anisotropic diffusion," IEEE Transactions on Biomedical Engineering, vol. 49, no. 9, pp. $997-$ 1014, 2002.

[18] J. R. Sanchez and M. L. Oelze, "An ultrasonic imaging speckle-suppression and contrast-enhancement technique by means of frequency compounding and coded excitation," IEEE Transactions on Ultrasonics, Ferroelectrics, and Frequency Control, vol. 56, no. 7, pp. 1327-1339, 2009.

[19] M. L. Oelze, "System and method for ultrasonic image processing," U.S. Patent 20090209858, 2009.

[20] M. L. Oelze, "Bandwidth and resolution enhancement through pulse compression," IEEE Transactions on Ultrasonics, Ferroelectrics, and Frequency Control, vol. 54, no. 4, pp. 768$781,2007$.

[21] T. Misaridis and J. A. Jensen, "Use of modulated excitation signals in medical ultrasound. Part I: basic concepts and expected benefits," IEEE Transactions on Ultrasonics, Ferroelectrics, and Frequency Control, vol. 52, no. 2, pp. 177-191, 2005.

[22] J. K. Tsou, J. Liu, and M. F. Insana, "Modeling and phantom studies of ultrasonic wall shear rate measurements using coded pulse excitation," IEEE Transactions on Ultrasonics, Ferroelectrics, and Frequency Control, vol. 53, no. 4, pp. 724734, 2006.

[23] J. R. Sanchez, M. Orescanin, and M. Oelze, "Improving image contrast using coded excitation for ultrasonic imaging," in Proceedings of the IEEE International Conference on Electro/Information Technology (EIT '10), pp. 1-6, Normal, Ill, USA, May 2010.

[24] C. P. Loizou, C. S. Pattichis, C. I. Christodoulou, R. S. H. Istepanian, M. Pantziaris, and A. Nicolaides, "Comparative evaluation of despeckle filtering in ultrasound imaging of the carotid artery," IEEE Transactions on Ultrasonics, Ferroelectrics, and Frequency Control, vol. 52, no. 10, pp. 1653-1669, 2005.

[25] Y. Yu and S. T. Acton, "Speckle reducing anisotropic diffusion," IEEE Transactions on Image Processing, vol. 11, no. 11, pp. 1260-1270, 2002.

[26] M. Nagao and T. Matsuyama, "Edge preserving smoothing," Computer Graphics and Image Processing, vol. 9, no. 4, pp. 394407, 1979.

[27] L. J. Busse, T. R. Crimmins, and J. R. Fienup, "A model based approach to improve the performance of the geometric filtering speckle reduction algorithm," in Proceedings of the IEEE Ultrasonics Symposium. Part 1, pp. 1353-1356, November 1995.

[28] P. Perona and J. Malik, "Scale-space and edge detection using anisotropic diffusion," IEEE Transactions on Pattern Analysis and Machine Intelligence, vol. 12, no. 7, pp. 629-639, 1990.

[29] P. M. Shankar, V. A. Dumane, C. W. Piccoli, J. M. Reid, F. Forsberg, and B. B. Goldberg, "Computer-aided classification of breast masses in ultrasonic B-scans using a multiparameter approach," IEEE Transactions on Ultrasonics, Ferroelectrics, and Frequency Control, vol. 50, no. 8, pp. 1002-1009, 2003.

[30] D. J. Sakrison, "On the role of observer and a distortion measure in image transmission," IEEE Transactions on Communications, vol. 25, no. 11, pp. 1251-1267, 1977.

[31] J. A. Jensen, "A model for the propagation and scattering of ultrasound in tissue," Journal of the Acoustical Society of America, vol. 89, no. 1, pp. 182-190, 1991.

[32] K. Raum and W. D. O’Brienjr Jr., "Pulse-echo field distribution measurement technique for high-frequency ultrasound sources," IEEE Transactions on Ultrasonics, Ferroelectrics, and Frequency Control, vol. 44, no. 4, pp. 810-815, 1997.
[33] S. Finn, M. Glavin, and E. Jones, "Echocardiographic speckle reduction comparison," IEEE Transactions on Ultrasonics, Ferroelectrics, and Frequency Control, vol. 58, no. 1, pp. 82-101, 2011.

[34] W. Wu, S. T. Acton, and J. Lach, "Real-time processing of ultrasound images with speckle reducing anisotropic diffusion," in Proceedings of the 40th Asilomar Conference on Signals, Systems, and Computers (ACSSC '06), pp. 1458-1464, November 2006. 

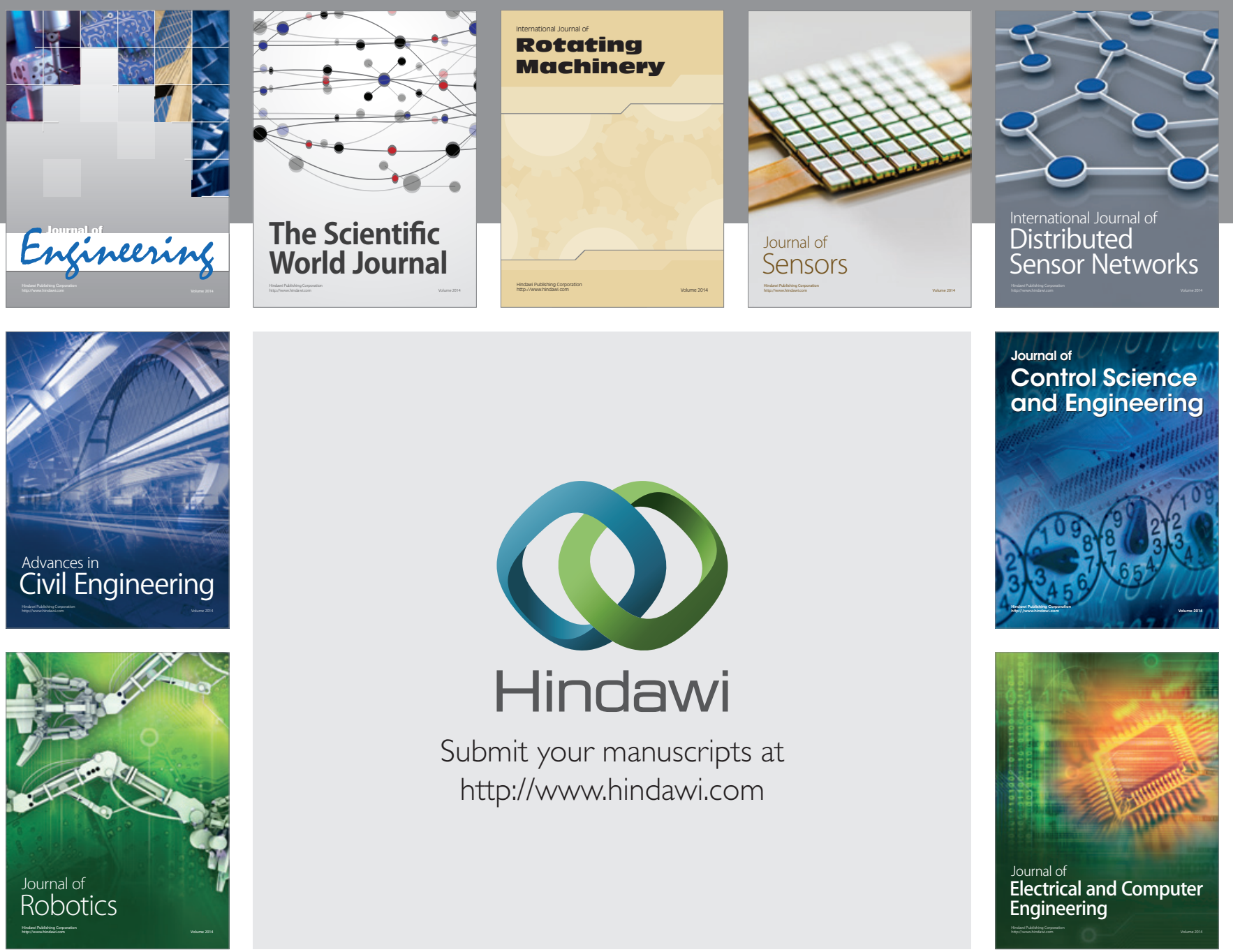

Submit your manuscripts at

http://www.hindawi.com
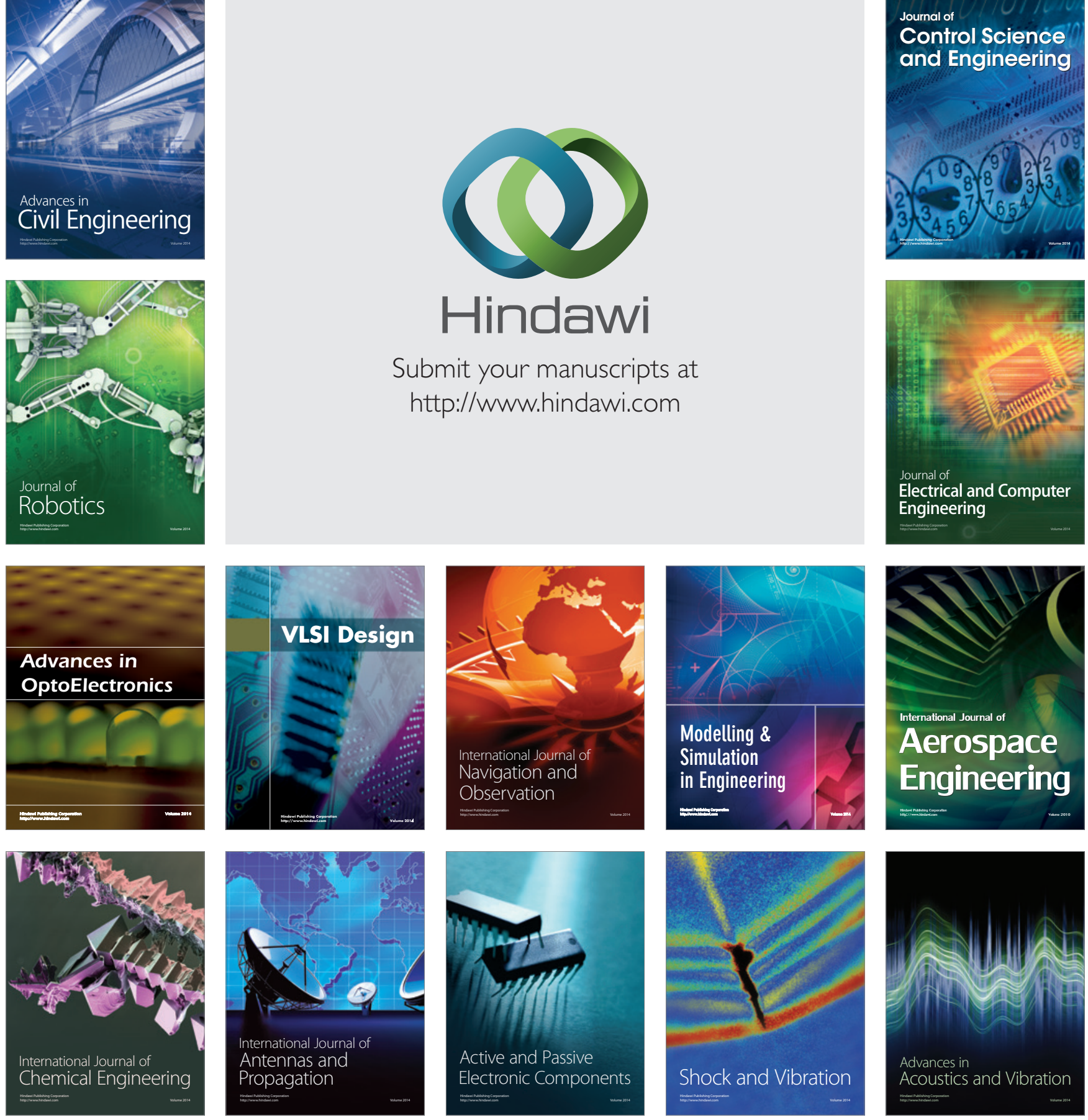